\title{
EXPLORACIÓN DE TIPOLOGÍAS HOTELERAS SEGÚN EL NIVEL DE IMPLEMENTACIÓN DE MEDIDAS DE AHORRO HÍDRICO A TRAVÉS DE UN ANÁLISIS CLÚSTER. EL CASO DE LLORET DE MAR (COSTA BRAVA $)^{1}$
}

\author{
Ariadna Gabarda-Mallorquí \\ Anna Ribas Palom \\ Universidad de Girona
}

\section{RESUMEN}

En los últimos años, las investigaciones sobre el consumo de agua en hoteles han prestado especial atención en relacionar esta variable con diferentes factores explicativos de tipo estructural. Sin embargo, los factores organizativos que pueden influir y modular el consumo de agua han sido poco explorados. A partir de la realización de encuestas a establecimientos hoteleros de Lloret de Mar (España), el presente trabajo tiene el objetivo de conocer el nivel de implementación actual de medidas de ahorro hídrico y de analizar los incentivos y barreras que perciben los gestores a la hora de aplicar nuevas medidas. A partir de un análisis clúster, los resultados indican que existen acusadas diferencias entre estos establecimientos en función de su grado de proactividad, sensibilización ambiental y tecnificación, y de las motivaciones y barreras que explican el nivel de implementación de medidas de ahorro hídrico.

Palabras clave: tipologías hoteleras; análisis clúster; medidas de ahorro hídrico; incentivos; barreras; Costa Brava.

Recibido: 17 de junio de 2016

Devuelto para su revisión: 21 de noviembre de 2016

Aceptado: 24 de abril de 2017

Departamento de Geografía e Instituto de Medio Ambiente.Universidad de Girona Plaza Ferrater Mora, 1. 17071 GIRONA (España).E-mail: ariadna.gabarda@udg.edu, anna.ribas@udg.edu

1 Este artículo se integra en el proyecto de investigación del Plan Nacional de I+D+I "Análisis de tendencias y estrategias de resiliencia en los usos y el consumo de agua en espacios turísticos. El caso de la Costa Brava" (CSO2013-41262-P) financiado por el Ministerio de Economía y Competitividad. 


\title{
Exploring hotel typologies according to water saving measures application through a cluster analysis. The case of Lloret de Mar (Costa Brava)
}

\begin{abstract}
During the last years, research on water consumption in hotels has been focused on the relation between this variable and other structural factors. However, the organizational elements that could modulate water consumption have been less explored. Through surveys conducted to hotel managers from Lloret de Mar (Spain), this study aims to know the level of the application of water-saving tools and to analyse the main incentives and obstacles perceived by hotel managers when implementing new water-savings mechanisms. Through a cluster analysis, the results show differences between the surveyed hotels depending on their level of proactivity, environmental awareness and technification, and the incentives and barriers that may explain the applied water-saving measures.
\end{abstract}

Keywords: hotel typologies; cluster analysis; water-saving measures; incentives; barriers; Costa Brava.

\section{INTRODUCCIÓN}

El agua es fundamental para las actividades económicas y el turismo no es una excepción (Cole, 2012), por lo que asegurar la disponibilidad de este recurso tanto en cantidad como en calidad se convierte en una cuestión primordial para el sector turístico. Los turistas necesitan agua para atender sus necesidades básicas pero también para la práctica de actividades deportivas, de ocio o salud como la natación, el golf o el recreo en parques acuáticos o spas (Gössling et al., 2012). También el agua es un recurso turístico desde el momento que se convierte en un elemento central del paisaje visitado por los turistas, desde los mismos jardines y espacios verdes de hoteles y campings hasta los espacios naturales como humedales o ambientes fluviales. Sin embargo, a escala local y especialmente en los momentos de máxima ocupación, la relación entre el turismo y el consumo de agua puede ser complejo y tener importantes implicaciones socioambientales. Las restricciones de agua o la mala calidad de la misma pueden afectar negativamente la imagen y la competitividad de los destinos tal como testimonian episodios de crisis hídricas que afectaron Benidorm (Rico-Amorós, Saurí, Olcina-Cantos, \& Vera-Rebollo, 2013) o Mallorca (Kent, Newnham, \& Essex, 2002). La probabilidad de que estos episodios se den cada vez con más frecuencia en destinos turísticos del litoral mediterráneo se espera que aumente en el futuro por una combinación de la reducción significativa de las precipitaciones como efecto esperable del cambio climático, un número creciente de visitantes y una mayor intensificación en el consumo de agua (Gössling et al., 2012; Kent et al., 2002).

A pesar de las cifras y la urgencia de reaccionar, son muchos los autores que han identificado importantes lagunas de conocimiento en las relaciones entre el turismo y el consumo de agua (Deyà \& Tirado, 2011; Hadjikakou, Chenoweth, \& Miller, 2013). Ello es especialmente preocupante desde el momento que el consumo de agua puede llegar a representar entre el $10 \%$ y el $20 \%$ del consumo total de agua potable en los países donde 
el turismo es un sector clave de la economía (Gössling et al., 2012). Este es el caso de España, donde, el turismo representa el 10,9\% del PIB, emplea al 11,9\% de la población activa (Instituto Nacional de Estadística, 2013) y es responsable de aproximadamente el $12 \%$ del consumo doméstico de agua (Gössling et al., 2012).

Sin embargo, y a escala de los establecimientos hoteleros, durante los últimos años empiezan a proliferar los trabajos que ponen de relieve la influencia del turismo en el consumo de agua y la necesidad de tener en cuenta diversos factores de tipo estructural (ej., categoría del establecimiento, número de plazas hoteleras, presencia de servicios con requerimientos hídricos elevados, como piscinas, spas o jardines, etc.) a la hora de determinar las estrategias necesarias para aumentar la resiliencia del sector turístico a los cambios en los patrones de la disponibilidad de agua derivados de los efectos del cambio climático (Barberán, Egea, Gracia-de-Rentería, \& Salvador, 2013; Bohdanowicz \& Martinac, 2007; Charara, Cashman, Bonnell, \& Gehr, 2011; Deyà \& Tirado, 2011). Sin embargo, los factores organizativos que pueden influir en el consumo de agua de los establecimientos hoteleros han sido menos explorados (Tzschentke, Kirk, \& Lynch, 2004). La adopción de medidas de ahorro hídrico contribuye sin duda alguna a un menor consumo de agua ya que permite, a partir de la implementación de mecanismos de distinto grado de tecnificación, un uso mucho más eficiente del agua. Existen múltiples razones que explican una mayor predisposición por parte del gestor hotelero (entiéndase director y/o propietario) a promover y llevar a cabo buenas prácticas ambientales (Quazi, Khoo, Tan, \& Wong, 2001), ya sean relacionadas con una mayor preocupación y sensibilización ambiental, con la intención de reducir costes operativos o con el obligado cumplimiento de las normativas ambientales, entre otras. De la misma manera, existen también obstáculos que no facilitan la aplicación de buenas prácticas ambientales de ahorro hídrico (Chan, 2008; Kasim, Gursoy, Okumus, \& Wong, 2014). Dichas barreras derivan de naturalezas distintas como la falta de recursos económicos y/o de tiempo, un bajo nivel de preocupación y sensibilización medioambiental o el poco convencimiento de los beneficios relacionados con el desarrollo de estas buenas prácticas ambientales (Kasim et al., 2014).

Es en este contexto que el presente trabajo se apoya en la realización de encuestas a los gestores de establecimientos hoteleros del municipio de Lloret de Mar (Costa Brava, España), con un doble objetivo. Por un lado, conocer el nivel de implementación actual de medidas de ahorro hídrico en los hoteles de esta destacada destinación turística del litoral mediterráneo y, por otro, analizar e interpretar los principales incentivos y barreras que explican el grado de aplicación de medidas de eficiencia hídrica en estos hoteles. Este trabajo se estructura en seis apartados. En el primero se destacan los principales incentivos y las barreras más comunes que explican el nivel de implementación de buenas prácticas ambientales en el sector hotelero. Seguidamente, se repasan brevemente cada una de las medidas de ahorro hídrico que, de acuerdo con el objetivo de este trabajo, permiten una reducción del consumo de agua y contribuyen a una mayor eficiencia en el uso de los recursos hídricos. En el tercer y cuarto apartados se presenta el área de estudio y la metodología empleada para la consecución de los objetivos planteados. Los resultados obtenidos se detallan en la quinta sección y se concluye el trabajo con la discusión de los mismos y unas breves conclusiones. 


\section{INCENTIVOS Y BARRERAS EXISTENTES EN EL SECTOR HOTELERO A LA IMPLEMENTACIÓN DE BUENAS PRÁCTICAS MEDIOAMBIENTALES}

A nivel general, un hotel puede llevar a cabo buenas prácticas ambientales a través de distintos mecanismos y/o procesos. Según Ayuso (2007), los más importantes desarrollados en el sector turístico a nivel internacional son los códigos de conducta, las buenas prácticas ambientales, las ecoetiquetas, los sistemas de gestión medioambiental (como la ISO 14001 o el EMAS) y los indicadores de gestión ambiental. Factores vinculados al nivel de sensibilización ambiental, la reducción de costes operativos o al grado de cumplimiento de las normativas ambientales, se convierten en elementos explicativos clave ya que determinan el nivel de adopción de buenas prácticas ambientales (O’Connor, Bord, Yarnal, \& Wiefek, 2002). Por ejemplo, un estudio desarrollado por el grupo hotelero alemán Steigenberger destaca que de la totalidad de los hoteles encuestados, un $20 \%$ tomaron medidas ambientales por razones económicas, otro $20 \%$ las implementan como respuesta a las demandas de los clientes y un $30 \%$ lo hicieron a fin de cumplir con los requerimientos normativos locales (Chan \& Wong, 2006). En este sentido, Kasim et al. (2014) añaden que los directivos de una empresa que emprenden acciones de responsabilidad social y ambiental pueden hacerlo también por razones de imagen y visibilidad. Otro ejemplo es el estudio llevado a cabo por Ayuso (2007) donde ha descrito y analizado los incentivos y las barreras percibidas por el sector hotelero español en el momento de emprender buenas prácticas ambientales. Los principales motivos que, según Ayuso, conducen a los gestores hoteleros a minimizar el impacto ambiental de su negocio radican tanto en querer ser más competitivos frente a las demandas de los propios clientes como en su propio nivel de sensibilización ambiental. En el caso de los sistemas de gestión medioambiental, éstos a menudo adoptan la forma de los estándares ya conocidos como EMAS y/o ISO 14001 (Chan \& Wong, 2006). A partir de un estudio aplicado en el sector hotelero de Hong Kong, Macau, Shenzhen y Guangzhou (China), Chan y Wong (2006) concluyeron que los motivos asociados a la decisión de adoptar una certificación ISO 14001 derivan principalmente de dos factores: la propia estrategia empresarial de la corporación y los requerimientos legales y normativos que los afectan.

Por otro lado, existen también barreras y obstáculos percibidos por los gestores hoteleros que no permiten la adopción de buenas prácticas ambientales (Chan, 2008). Dichas barreras pueden ser de dos tipos. Por una parte, barreras internas, como la falta de personal especializado para su adopción, el desconocimiento relativo a los beneficios derivados de la implementación de buenas prácticas ambientales o la simple resistencia al cambio. Por otra parte, barreras externas, como los costes económicos asociados a su implementación o la falta de un marco normativo claro y estricto (Hillary, 2004). Entre los estudios que han constatado la existencia de estas y otros tipos de barreras destaca el de Ayuso (2007) para el caso español, en el que constata el elevado coste de instalación y mantenimiento de los sistemas e infraestructuras necesarios para reducir el impacto ambiental, especialmente en lo que se refiere a la renovación de las certificaciones ambientales, la falta de tiempo y/o conocimiento técnico por parte de los gestores hoteleros, el riesgo de poner en peligro la satisfacción del cliente, la dificultad para involucrar al personal empleado y/o la percepción de que el sector hotelero no es el responsable de los impactos medioambientales que pueda ocasionar. En un estudio desarrollado en una muestra representativa de hoteles 
de Hong Kong, Chan (2008) ha determinado que la primera gran barrera percibida por los gestores hoteleros que no permite adoptar un sistema de gestión medioambiental es la relacionada con los altos costes de implementación y mantenimiento del propio sistema de gestión. El segundo principal obstáculo es la falta de asesoramiento técnico, seguido de la falta de conocimientos y habilidades de los propios gestores para su implementación.

A modo de síntesis, y con el objetivo de explorar algunos factores explicativos según su naturaleza, la tabla 1 muestra distintos incentivos y barreras percibidos por los gestores hoteleros cuando se trata de implementar buenas prácticas ambientales y que han sido explorados en estudios anteriores. Para el presente trabajo, estos factores pueden resumirse en cuatro grandes grupos: los instrumentales y/o normativos, los socioculturales, los económicos y los estratégicos y/o empresariales (tabla 1). El primer factor reúne aquellos incentivos o barreras relacionados con la legislación competente en materia ambiental y los instrumentos externos gracias a los cuáles los gestores hoteleros pueden recibir más (o menos) soporte cuando se trata de llevar a cabo medidas de gestión sostenibles. El factor sociocultural va íntimamente relacionado con el nivel de sensibilización y preocupación del propio gestor hotelero hacia los problemas ambientales actuales y la propia convicción y voluntad por intentar minimizarlos. El tercer factor, el económico, agrupa todos aquellos incentivos y/o barreras que vienen determinados por cuestiones económicas tanto a nivel de costes como de beneficios del propio hotel. El último factor es el estratégico y empresarial y reúne los incentivos y barreras derivados de cuestiones corporativas de la empresa, como la propia gestión empresarial y/o laboral, la publicidad y el márquetin, entre otros factores.

\section{Tabla 1 \\ SÍNTESIS DE LOS INCENTIVOS Y BARRERAS \\ QUE EXPLICAN LA ADOPCIÓN DE MEDIDAS MEDIOAMBIENTALES \\ IDENTIFICADOS POR LOS GESTORES HOTELEROS DE DISTINTAS DESTINACIONES TURÍSTICAS}

\begin{tabular}{|c|c|c|}
\hline & Localización & Referencia \\
\hline \multicolumn{3}{|l|}{ Incentivos } \\
\hline \multicolumn{3}{|l|}{ Factores instrumentales y/o normativos } \\
\hline Subvenciones y ayudas & Singapur & (Chan \& Wong, 2006) \\
\hline \multirow{2}{*}{$\begin{array}{l}\text { Obligado cumplimiento en la legislación nacional } \\
\text { y/o regional }\end{array}$} & Austria & (Chan \& Wong, 2006) \\
\hline & España & (Ayuso, 2007) \\
\hline \multicolumn{3}{|l|}{ Factores socioculturales } \\
\hline \multirow[t]{4}{*}{$\begin{array}{l}\text { Preocupación e intención de reducir el impacto } \\
\text { medioambiental de la empresa }\end{array}$} & Oeste de Australia & $\begin{array}{c}\text { (Carlsen, Getz, \& Ali-Knight, } \\
\text { 2001) }\end{array}$ \\
\hline & Europa & (Bohdanowicz, 2005) \\
\hline & España & (Ayuso, 2007) \\
\hline & Hong Kong, China & (Chan \& Hawkins, 2010) \\
\hline \multirow[t]{3}{*}{ Es de obligación ética y moral } & Oeste de Australia & (Carlsen et al., 2001) \\
\hline & $\begin{array}{l}\text { Escocia, Reino } \\
\text { Unido }\end{array}$ & (Tzschentke et al., 2004) \\
\hline & Dinamarca & (Bramwell \& Alletorp, 2001) \\
\hline
\end{tabular}




\begin{tabular}{|c|c|c|}
\hline \multicolumn{3}{|l|}{ Factores económicos } \\
\hline \multirow[t]{6}{*}{ Reducción de costes } & $\begin{array}{l}\text { Escocia, Reino } \\
\text { Unido }\end{array}$ & (Tzschentke et al., 2004) \\
\hline & Dinamarca & (Bramwell \& Alletorp, 2001) \\
\hline & Europa & (Bohdanowicz, 2005) \\
\hline & $\begin{array}{l}\text { Londres, Reino } \\
\text { Unido }\end{array}$ & $\begin{array}{l}\text { (Knowles, Macmillan, Palmer, } \\
\text { Grabowski, \& Hashimoto, 1999) }\end{array}$ \\
\hline & España & (Ayuso, 2007) \\
\hline & Hong Kong, China & (Chan \& Hawkins, 2010) \\
\hline \multicolumn{3}{|l|}{ Factores estratégicos y/o empresariales } \\
\hline \multirow[t]{2}{*}{ Respuesta a las demandas de los clientes } & Europa & (Bohdanowicz, 2005) \\
\hline & España & (Ayuso, 2007) \\
\hline \multirow[t]{4}{*}{ Mejora de la imagen del hotel } & Europa & (Bohdanowicz, 2005) \\
\hline & España & (Ayuso, 2007) \\
\hline & Hong Kong, China & (Chan \& Hawkins, 2010) \\
\hline & $\begin{array}{l}\text { Edimburgo, Reino } \\
\text { Unido }\end{array}$ & (Kirk, 1998) \\
\hline \multirow[t]{2}{*}{ Mejora de la competitividad en el mercado } & $\begin{array}{l}\text { Escocia, Reino } \\
\text { Unido } \\
\end{array}$ & (Tzschentke et al., 2004) \\
\hline & Dinamarca & (Bramwell \& Alletorp, 2001) \\
\hline $\begin{array}{l}\text { Mejora de la organización interna de la propia } \\
\text { empresa }\end{array}$ & España & (Ayuso, 2007) \\
\hline $\begin{array}{l}\text { Corrección de conductas poco sostenibles por } \\
\text { parte de los clientes }\end{array}$ & $\begin{array}{l}\text { Escocia, Reino } \\
\text { Unido }\end{array}$ & (Tzschentke et al., 2004) \\
\hline \multicolumn{3}{|l|}{ Barreras } \\
\hline \multicolumn{3}{|l|}{ Factores instrumentales y/o normativos } \\
\hline \multirow[t]{3}{*}{$\begin{array}{l}\text { Inexistencia de legislación y/o subvenciones que } \\
\text { estimulen buenas prácticas medioambientales }\end{array}$} & $\begin{array}{c}\text { Isla de Wight, Reino } \\
\text { Unido }\end{array}$ & (Hjalager, 1998) \\
\hline & Mallorca, España & (Hjalager, 1998) \\
\hline & $\begin{array}{l}\text { Condado de East } \\
\text { Sussex, Reino } \\
\text { Unido } \\
\end{array}$ & (Berry \& Ladkin, 1997) \\
\hline $\begin{array}{l}\text { No existen compensaciones económicas que } \\
\text { premien las buenas prácticas ambientales }\end{array}$ & Dinamarca & (Bramwell \& Alletorp, 2001) \\
\hline $\begin{array}{l}\text { Limitado acceso a guías, folletos informativos,... } \\
\text { sobre buenas prácticas ambientales }\end{array}$ & $\begin{array}{l}\text { Grandes ciudades, } \\
\text { Reino Unido }\end{array}$ & (Hobson \& Essex, 2001) \\
\hline $\begin{array}{l}\text { Desconocimiento acerca de las posibles buenas } \\
\text { prácticas ambientales a implementar }\end{array}$ & Europa & (Bohdanowicz, 2005) \\
\hline \multirow{3}{*}{$\begin{array}{l}\text { Falta de organizaciones y/o asociaciones } \\
\text { empresariales que estimulen las innovaciones } \\
\text { medioambientales }\end{array}$} & $\begin{array}{c}\text { Isla de Wight, Reino } \\
\text { Unido } \\
\end{array}$ & (Hjalager, 1998) \\
\hline & $\begin{array}{l}\text { Bornholm, } \\
\text { Dinamarca }\end{array}$ & (Hjalager, 1998) \\
\hline & España & (Ayuso, 2007) \\
\hline
\end{tabular}




\begin{tabular}{|c|c|c|}
\hline \multicolumn{3}{|l|}{ Factores socioculturales } \\
\hline \multirow[t]{2}{*}{$\begin{array}{l}\text { Desconocimiento o minimización del impacto } \\
\text { medioambiental provocado por el sector turístico }\end{array}$} & $\begin{array}{c}\text { Condado de East } \\
\text { Sussex, Reino } \\
\text { Unido } \\
\end{array}$ & (Berry \& Ladkin, 1997) \\
\hline & Reino Unido & (Forsyth, 1997) \\
\hline $\begin{array}{l}\text { Complejidad asociada a las buenas prácticas } \\
\text { ambientales }\end{array}$ & $\begin{array}{c}\text { Condado de East } \\
\text { Sussex, Reino } \\
\text { Unido }\end{array}$ & (Berry \& Ladkin, 1997) \\
\hline \multirow[t]{2}{*}{ Bajo nivel de responsabilidad socioambiental } & Bailía de Guernsey & (Stabler \& Goodall, 1997) \\
\hline & $\begin{array}{l}\text { Cornwall, Reino } \\
\text { Unido }\end{array}$ & $\begin{array}{l}\text { (Vernon, Essex, Pinder, \& Curry, } \\
\text { 2003) }\end{array}$ \\
\hline \multirow[t]{2}{*}{ Falta de tiempo } & $\begin{array}{l}\text { Grandes ciudades, } \\
\text { Reino Unido }\end{array}$ & (Hobson \& Essex, 2001) \\
\hline & $\begin{array}{l}\text { Condado de East } \\
\text { Sussex, Reino } \\
\text { Unido }\end{array}$ & (Berry \& Ladkin, 1997) \\
\hline \multirow[t]{3}{*}{ Falta de conocimiento técnico } & $\begin{array}{l}\text { Grandes ciudades, } \\
\text { Reino Unido }\end{array}$ & (Hobson \& Essex, 2001) \\
\hline & Hong Kong, China & (Chan, 2008) \\
\hline & España & (Ayuso, 2007) \\
\hline \multicolumn{3}{|l|}{ Factores económicos } \\
\hline \multirow[t]{6}{*}{$\begin{array}{l}\text { Elevada inversión inicial para la implementación } \\
\text { de buenas prácticas ambientales }\end{array}$} & $\begin{array}{l}\text { Cornwall, Reino } \\
\text { Unido }\end{array}$ & (Vernon et al., 2003) \\
\hline & Dinamarca & (Bramwell \& Alletorp, 2001) \\
\hline & Bailía de Guernsey & (Stabler \& Goodall, 1997) \\
\hline & $\begin{array}{l}\text { Grandes ciudades, } \\
\text { Reino Unido }\end{array}$ & (Hobson \& Essex, 2001) \\
\hline & Hong Kong, China & (Chan, 2008) \\
\hline & España & (Ayuso, 2007) \\
\hline \multirow[t]{2}{*}{$\begin{array}{l}\text { Bajo margen de beneficio económico asociado a } \\
\text { las prácticas ambientales }\end{array}$} & $\begin{array}{c}\text { Cornwall, Reino } \\
\text { Unido }\end{array}$ & (Vernon et al., 2003) \\
\hline & Dinamarca & (Bramwell \& Alletorp, 2001) \\
\hline \multicolumn{3}{|l|}{ Factores estratégicos y/o empresariales } \\
\hline \multirow[t]{2}{*}{ No es valorado por los clientes } & $\begin{array}{l}\text { Cornwall, Reino } \\
\text { Unido }\end{array}$ & (Vernon et al., 2003) \\
\hline & Reino Unido & (Forsyth, 1997) \\
\hline Poca colaboración por parte de los clientes & España & (Ayuso, 2007) \\
\hline Dificultades para involucrar al personal empleado & España & (Ayuso, 2007) \\
\hline
\end{tabular}

\section{INICIATIVAS DE AHORRO HÍDRICO EN HOTELES}

Las iniciativas de ahorro hídrico existentes actualmente en el sector turístico abarcan un gran número de medidas implementadas con distintos niveles tanto de tecnificación como de inversión económica. En la tabla 2 se presentan medidas de ahorro hídrico aplicadas en algunos establecimientos hoteleros. 


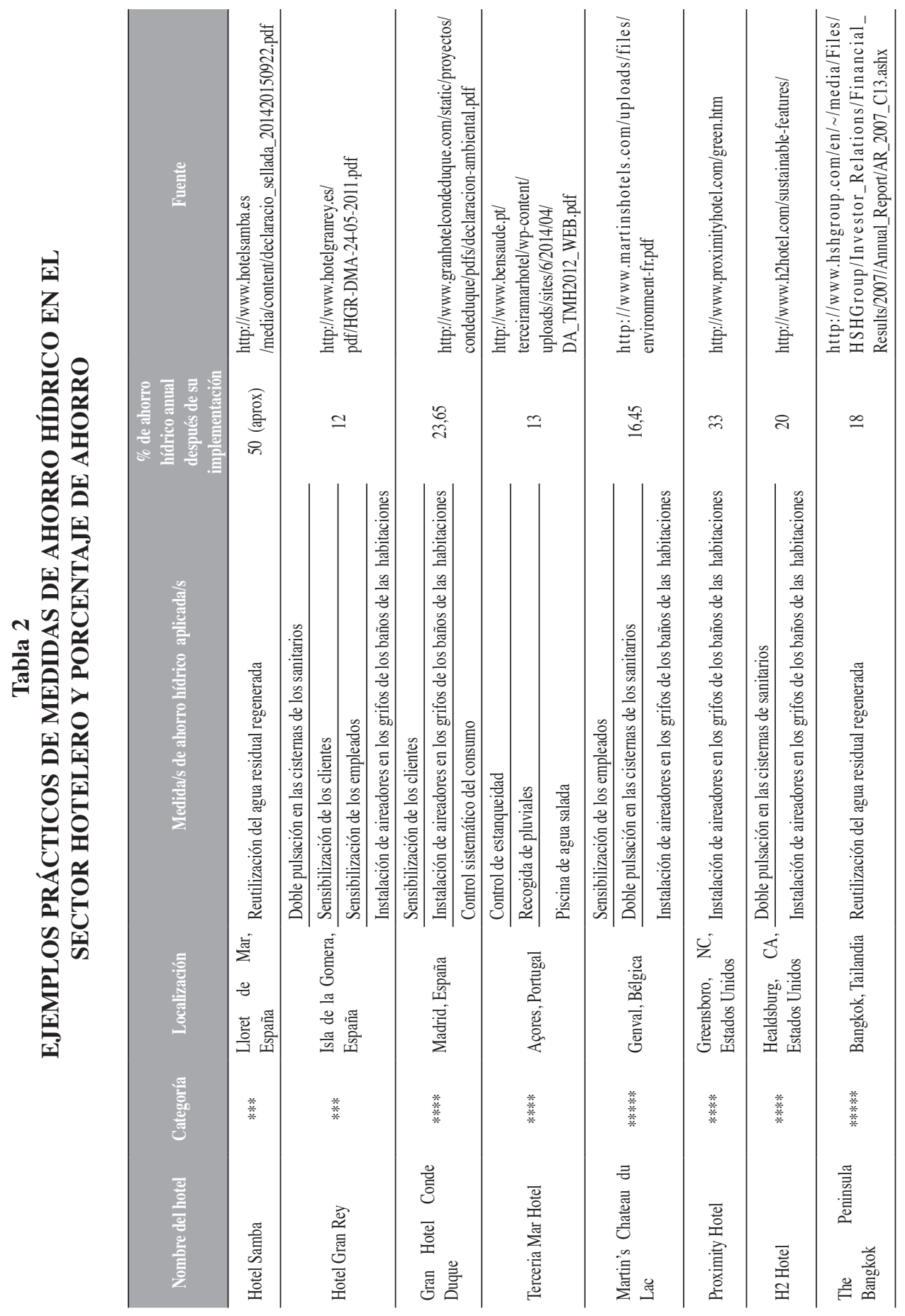


Todas estas y otras medidas se pueden agrupar en dos grandes categorías (medidas básicas y avanzadas).

\subsection{Medidas básicas}

Son aquellas iniciativas que requieren de una intencionalidad en su implementación por parte de los gestores hoteleros pero que no implican una gran complejidad técnica ni, en general, una elevada inversión económica. Es el caso de la instalación de aireadores, sensores y/o temporizadores en grifos y duchas, los dobles pulsadores en las cisternas de los sanitarios, el control de lavado y uso de programas de ahorro en lavadoras, el diseño de jardines con vegetación autóctona, la pavimentación de una parte o todas las zonas exteriores, el control detallado del consumo de agua, la sensibilización y motivación hacia el ahorro hídrico a los clientes y a los empleados y la sustitución de electrodomésticos con elevados requerimientos de agua por otros más eficientes.

\subsubsection{Aireadores, sensores y temporizadores en grifos y duchas}

La instalación de este tipo de mecanismos en los baños de las habitaciones permite un ahorro de agua considerable (Gatt \& Schranz, 2015; Gössling et al., 2012). Tanto los aireadores en los grifos de los lavamanos y de las duchas como los sensores de movimiento y los temporizadores instalados en todo tipo de grifos, permiten un control del consumo hídrico y evitan también las pérdidas por goteo. Los aireadores en los grifos de los lavamanos suponen un ahorro del 50\% del consumo, pasando de un consumo aproximado de 12 litros por minuto a 6 (Smith, Hargroves, Desha, \& Stasinopoulos, 2009). El coste de su implementación dependerá básicamente de si es necesario reemplazar el grifo en su totalidad o si se pueden adaptar aireadores a los grifos ya existentes, instalando únicamente el mecanismo ahorrador. De aquí que el coste estimado de esta medida puede variar entre 10 y 200 euros por unidad (Styles, Schoenberger, \& Galvez-Martos, 2015). Sin embargo, y a pesar de que su eficiencia ha sido probada, los mecanismos automáticos en los grifos pueden alcanzar incluso una mayor eficiencia si los usuarios son sensibles al ahorro y muestran comportamientos realmente sostenibles en relación a sus hábitos de consumo de agua (Hills, Birks, \& McKenzie, 2002). Cada vez son más numerosos los países en los que los aireadores en grifos son de obligatoria instalación en aquellos hoteles de nueva construcción (Gabarda-Mallorquí \& Ribas, 2016; Manco Silva, Erazo, \& Ocampo Cruz, 2012).

\subsubsection{Sistemas de descarga de doble pulsación en sanitarios}

Generalmente, las cisternas de los sanitarios tienen una capacidad de almacenaje de agua de entre 6 y 9 litros. La doble pulsación en sanitarios permite al usuario poder escoger la descarga de agua de manera que se vacíe por completo la cisterna o bien se descargue solo la mitad (Soriano, 2010). Con este sistema, se puede alcanzar un ahorro del $45 \%$ en el consumo de agua destinado a los inodoros (Manco, Erazo, \& Ocampo, 2012). El coste de las cisternas con doble pulsador se sitúa alrededor de los 200 euros. A pesar del ahorro de agua que comporta la instalación de mecanismos de control de descarga, el 
componente usuario es vital para la correcta utilización de los pulsadores. El desconocimiento o mala praxis del usuario hace invisible la eficiencia de este tipo de dispositivo.

\subsubsection{Control de lavado y programas de ahorro en lavadoras}

Actualmente existen lavadoras con múltiples programas de lavado, algunos de los cuales permiten reducir el consumo de agua hasta 80 litros por lavado (Manco et al., 2012). En muchos casos, los hoteles que disponen de su propia lavandería funcionan con maquinaria industrial como lavadoras con gran capacidad de carga y funciones de lavado llamados "eco" que permiten un ahorro considerable de agua. Styles et al. (2015) constatan que externalizando el servicio de lavandería a empresas especializadas se reduce a 0 el consumo derivado de las lavadoras en el hotel. Existen empresas de lavandería que cuentan con equipamientos altamente tecnificados y/o con certificaciones ambientales, con lo que el consumo de agua se optimiza mucho más.

\subsubsection{Diseño de jardines y zonas verdes con vegetación autóctona}

La xerojardinería engloba los conocimientos y técnicas más adecuadas para el diseño y gestión de jardines en zonas cálidas, como el Mediterráneo, y que permiten un uso más eficiente de agua. Por ejemplo, el diseño de zonas verdes con vegetación autóctona permite reducir el consumo de agua destinado al riego de las plantas ornamentales ya que, especialmente en la zona mediterránea, se evitan los jardines con césped o plantas con alto requerimiento hídrico (Deyà \& Tirado, 2011). Así, las plantas crasas con alta resistencia al estrés hídrico son muy convenientes en jardines de hoteles de regiones cálidas (Smith et al., 2009). A pesar de ello, hay que tener en cuenta que el diseño de jardines con especies vegetales exóticas y resistentes al estrés hídrico puede aumentar el riesgo de invasión biológica (Padullés, Vila, \& Barriocanal, 2015). La adopción de técnicas asociadas a la xerojardinería puede llegar a suponer un ahorro hídrico anual del 33\% del total de agua consumida (Padullés, 2015).

\subsubsection{Pavimentación de una parte o todos los espacios exteriores}

Esta medida es sin duda drástica pero efectiva ya que se reduce o incluso se elimina en casi su totalidad el consumo de agua en las zonas exteriores de los hoteles. De hecho, en el ámbito del turismo residencial es uno de los cambios más importantes que se están constatando en los últimos años en los exteriores de las segundas residencias del litoral mediterráneo peninsular (Morote \& Hernández, 2014).

\subsubsection{Control detallado del consumo de agua}

Una planificación adecuada de la gestión del agua del propio establecimiento hotelero permite controlar cualquier disfunción en el sistema de suministro y distribución del agua (Styles et al., 2015). Así, con el control detallado del consumo, ya sea a partir del registro de facturación o la lectura de contadores y subcontadores, se puede actuar con mucha más rapidez ante cualquier anomalía detectada. Los controles periódicos de este tipo efectuados 
en los hoteles de la cadena hotelera sueca Scandic permitieron una reducción acumulada del $25 \%$ del consumo de agua a partir del momento en el que llevaron a cabo estrictos planes de control hídrico (Styles et al., 2015).

\subsubsection{Información y sensibilización a los clientes sobre el ahorro hídrico}

Es una de las medidas de ahorro hídrico más implementadas en hoteles y edificios públicos. A partir, por ejemplo, de carteles en los baños de las habitaciones instando al cliente a reutilizar sus toallas más de una vez, se consigue reducir las cargas en la lavandería y con ello el consumo de agua. Para ello, conviene asegurar la presencia de colgadores de ropa en los baños a fin de facilitar esta práctica (Smith et al., 2009).

\subsubsection{Sensibilización de los empleados sobre el ahorro hídrico}

El personal empleado de un hotel juega un rol importante en el consumo hídrico y energético. En ámbitos como la cocina, la limpieza de las habitaciones, la limpieza general y el riego de los espacios exteriores y las zonas acuáticas, los trabajadores pueden adoptar una actitud proactiva en las tareas cuotidianas y contribuir al ahorro de agua del establecimiento. Por ejemplo, las buenas prácticas hídricas en las cocinas (Gössling et al., 2012), como evitar la descongelación de alimentos bajo el agua del grifo (Manco et al., 2012) o poner en marcha los lavaplatos solo cuando estén llenos (Smith et al., 2009), se consigue reducir notablemente el consumo de agua.

\subsubsection{Sustitución de electrodomésticos de alto consumo por otros de más eficientes}

La sustitución de los electrodomésticos por otros más eficientes desde un punto de vista hídrico es también una buena medida de ahorro. Existen en el mercado múltiples opciones que permiten reducir el gasto de agua con el mismo servicio y las mismas prestaciones que los electrodomésticos tradicionales. Las lavadoras con etiqueta de eficiencia energética $\mathrm{A}^{+++}$, por ejemplo, consumen un $60 \%$ menos de agua que aquellas que no presentan controles de eficiencia (Smith et al., 2009). La reposición de electrodomésticos es, pues, una gran oportunidad para adquirir nuevos aparatos con una elevada eficiencia tanto hídrica como energética. Los hoteles participantes en el programa SHINE, proyecto impulsado por la School of Public Health of Harvard College y que tiene por objetivo asesorar a organismos y empresas de cualquier sector económico para reducir su impacto ambiental, ahorraron cerca de $1.000 \mathrm{~m}^{3}$ anuales con la aplicación de diversas medidas, siendo una de las más relevantes el cambio de sus lavadoras (Alexander \& Kennedy, 2002).

\subsection{Medidas avanzadas}

Por último, una segunda categoría de medidas de ahorro hídrico agrupa aquellas que implican una clara intencionalidad de aplicación por parte del mismo gestor del hotel y que, en general, requieren de una importante inversión económica inicial. Son medidas que precisan de un conocimiento avanzado y un asesoramiento técnico para poderlas llevar a 
cabo. Es el caso de la implementación de un sistema de control de estanqueidad en la red de suministro y distribución del agua, la optimización de los sistemas de riego en jardines, la reutilización de las aguas grises, la recogida y uso de aguas pluviales, la sustitución de los equipos de refrigeración por equipos de condensación de aire, la instalación de reguladores de presión en las tomas de agua principales, la instalación de piscinas con agua de mar y el cubrimiento de las piscinas exteriores a fin de reducir la evaporación del agua.

\subsubsection{Control de estanqueidad en la red de abastecimiento y distribución del agua}

Las fugas y pérdidas de agua, tanto a través de la red interna de abastecimiento y distribución como en los mismos dispositivos hídricos (grifería, sistemas de filtraje en las piscinas, etc) pueden representar un importante aumento en el consumo total de agua del hotel. Controlar la estanqueidad de todo el sistema de abastecimiento y distribución interno y detectar las pérdidas es clave para el ahorro de agua. Se calcula que un grifo en mal estado puede llegar a perder entre 30 y 700 litros por día (Manco et al., 2012). La instalación de subcontadores en las principales entradas de agua del propio establecimiento permite un control exhaustivo del consumo total y detectar con facilidad cualquier variación no deseada.

\subsubsection{Optimización de los sistemas de limpieza y riego en zonas verdes y jardines}

La limpieza y riego de zonas verdes y jardines puede suponer una parte importante del consumo de agua total de un hotel. A pesar de las diferencias entre regiones debido a las particularidades climáticas, las características del suelo y de la vegetación y/o la eficiencia en los sistemas de riego (Gössling et al., 2012), el volumen de agua destinado al riego de jardines puede suponer hasta el 50\% del total consumido (Gössling, 2001). Sin embargo, existen sistemas y mecanismos de irrigación que permiten un riego mucho más eficiente. El riego de jardines por goteo es una de las técnicas que reemplaza las técnicas más tradicionales (Kasim et al., 2014) como el riego con manguera o manual. Aunque el riego por goteo es una de las técnicas más eficientes (entre un 75 y un 90\% de eficiencia) (Fuentes, 2003), en el caso de los jardines con césped presentes en muchos hoteles no es la más adecuada (Parés, Domene, \& Saurí, 2004). Uno de los mecanismos de riego con una aplicación creciente en hoteles son los difusores en mangueras de riego similares a los aspersores pero con un alcance menor. Otros sistemas de optimización hídrica en el riego de jardines y zonas verdes consiste en la instalación de sistemas de programación automática del riego en función de las condiciones meteorológicas, práctica ésta bastante extendida en el caso del cuidado y mantenimiento de los campos de golf (Gössling et al., 2012). Aunque sea menos eficiente, el riego por aspersión puede optimizarse controlando que el agua no caiga en zonas pavimentadas y evitando regar durante las horas de máxima insolación o en días con mucho viento (Smith et al., 2009).

\subsubsection{Reutilización de aguas grises}

Las aguas grises que provienen de los baños de las habitaciones pueden ser tratadas y reutilizadas a través de las cisternas de los sanitarios o bien para el riego de los jardines y zonas verdes. Es un sistema técnicamente viable que requiere de una fuerte inversión inicial (Smith et al., 2009) pero que se convierte en un importante recurso hídrico alternativo (Antakyali, 
Krampe, \& Steinmetz, 2008). En hoteles de nueva construcción, la instalación de sistemas para la reutilización de agua residual regenerada es una gran oportunidad para contribuir a un menor consumo de agua potable (Smith et al., 2009). El uso de agua residual regenerada a partir de las aguas grises es una técnica muy avanzada que cuenta con pocos pero interesantes ejemplos de aplicación en el sector turístico (Gössling et al., 2012). En este sentido se pueden encontrar casos concretos de establecimientos de alojamiento turístico donde su eficiencia ha sido demostrada, como el Kühlungsborn Camping en Alemania (Styles et al., 2015) o el Hotel Samba en España (Gabarda-Mallorquí \& Ribas, 2016).

\subsubsection{Recogida y reutilización de aguas pluviales}

Algunos estudios apuntan que el uso del agua pluvial o nival puede reducir el consumo total de un hotel en un 55\% con una inversión económica relativamente baja (Kasim et al., 2014). La recogida de agua de lluvia es muy adecuada para hoteles situados en regiones con elevados índices de precipitación y puede contribuir a reducir el riesgo de inundación, especialmente en sótanos y aparcamientos subterráneos (Smith et al., 2009). A pesar de todo, las normativas urbanísticas y ambientales municipales son los entes reguladores clave de este tipo de sistemas y por ello su posible potenciación se verá condicionada por la consideración que tengan en las normativas ambientales locales, regionales y/o nacionales implicadas.

\subsubsection{Sustitución de los equipos de refrigeración por condensación de agua por equipos de condensación de aire}

Los equipos de refrigeración por condensación de agua son usuales en edificios de regiones cálidas, puesto que resultan muy útiles cuando la temperatura del aire oscila entre 30 y $45^{\circ} \mathrm{C}$, pero consumen agua (Jiménez, 2011). Los sistemas de condensación de aire no precisan de agua para su correcto funcionamiento por lo que permiten reducir el consumo de agua del hotel. Sin embargo, los equipos de condensación de aire tienen el inconveniente que consumen entre un 30 y un 50\% más de energía que los de agua incluso refrigerando menos que los equipos que condensan con agua (Jiménez, 2011).

\subsubsection{Instalación de reguladores de presión}

La reducción de la presión de agua en el circuito de suministro y distribución del agua también es una medida que contribuye a un menor consumo hídrico. Los reguladores de presión instalados en la toma de agua principal del hotel permiten regular la presión y estabilizarla permanentemente, reduciendo el riesgo de daños en las tuberías y las consecuentes pérdidas incontroladas de agua. El valor de referencia de la presión del sistema para conseguir una mayor eficiencia hídrica debe ser menor a 3 bares.

\subsubsection{Instalación de piscinas saladas}

Las piscinas saladas son también un recurso que puede convertirse en una fuente hídrica alternativa que reduce ostensiblemente el consumo total de agua del un hotel. En este caso hay que diferenciar entre dos tipos de instalaciones: las piscinas con agua de mar 
y las de agua salada. En el primer caso el suministro de agua proviene directamente del mar con lo que se consigue substituir en su totalidad el consumo de agua potable de la red de suministro, mientras que en el segundo el llenado de piscinas proviene de agua potable de red con un tratamiento de cloración salina. En este último caso, el vaciado y llenado de la piscina tiende a ser menos frecuente en comparación con las piscinas convencionales.

\subsubsection{Cubrimiento de piscinas para reducir la evaporación de agua}

La evaporación de agua en piscinas exteriores situadas en climas cálidos constituye el principal motivo de pérdida de agua en este tipo de instalaciones (Randolph \& Troy, 2008). El cubrimiento de las piscinas de los hoteles durante las horas de menos afluencia de usuarios, como la noche, puede reducir ostensiblemente la evaporación del agua (Gössling et al., 2012). Con este tipo de mecanismos se puede llegar a ahorrar hasta el 50\% del consumo de agua asociado al proceso de reposición de agua en las piscinas (Styles et al., 2015). En el ámbito del turismo residencial el cubrimiento de las piscinas exteriores también se recomienda como medida eficaz a fin de reducir el consumo de agua de las viviendas (Hof \& Schmitt, 2011).

\section{4. ÁREA DE ESTUDIO}

Lloret de Mar (Girona), municipio turístico de la Costa Brava (figura 1), tiene una extensión de 48,7 km² y, con datos de 2014, una población de 38.624 habitantes censados y 55.037 habitantes equivalentes a tiempo completo anual (ETCA). Con cerca de 30.000 plazas hoteleras, concentra más del $45 \%$ de las existentes en el litoral de la Costa Brava. Durante el año 2015 visitaron el municipio 1.119.294 turistas, la mayor parte de ellos $(40,84 \%)$ durante los meses de junio, julio y agosto. De acuerdo con los datos publicados por el Instituto Nacional de Estadística, en 2015 Lloret de Mar fue el segundo municipio catalán con mayor número de visitantes después de la ciudad de Barcelona. De hecho, el municipio se ha especializado en el alojamiento hotelero desde sus inicios como destinación turística internacional. La evolución de la categoría de la planta hotelera ha variado mucho a lo largo de los últimos 50 años. En un primer momento, durante el inicio del boom turístico en la década de los años cincuenta, muchas tabernas se convirtieron en hostales y pequeños hoteles para alojar una cantidad creciente de visitantes. De esta manera, los hostales y pensiones, así como los hoteles de una, dos y tres estrellas, conformaban la mayor parte de la oferta hotelera de esta destinación turística. A partir de la década de los noventa, el desarrollo del Plan Marco de Competitividad del Turismo Español (1992-1995) permitió la modernización de la planta hotelera lo que facilitó que los hoteles de mayor categoría ganaran cuota en detrimento del resto (Gabarda-Mallorquí $\&$ Ribas, 2016). Actualmente, los hoteles de tres $(33,06 \%)$ y cuatro estrellas $(25 \%)$ son los más numerosos, seguidos de los hostales y pensiones $(20,97 \%)$, de los hoteles de una $(9,67 \%)$ y dos estrellas $(8,06 \%)$ y de los de cinco estrellas $(3,23 \%)$. Teniendo en cuenta el número de plazas hoteleras, los hostales y pensiones $(3,88 \%)$ y los hoteles de cinco estrellas $(2,65 \%)$ son los grupos menos numerosos, mientras que los hoteles de tres y cuatro estrellas siguen siendo las categorías con más plazas disponibles reuniendo el $82,71 \%$ del total de plazas hoteleras existentes. 
Lloret de Mar se abastece principalmente del agua de la estación de tratamiento de agua potable (ETAP) de Tossa-Lloret, ubicada en el vecino municipio de Tordera (sur de la Costa Brava) y gestionada por el Consorcio de la Costa Brava. Desde 2002 también se abastece de la planta desalinizadora de la Tordera (Instalación de Tratamiento de Agua Marina, ITAM), ubicada en Blanes, con capacidad para aportar 2,5 hm³/año a los municipios de Lloret de Mar y Tossa de Mar. También en verano de 2007 entró en servicio la columna vertebral de la red de agua regenerada de Lloret que distribuye los caudales regenerados de la estación depuradora de aguas residuales (EDAR) del mismo municipio, con el objetivo de aportar agua no potable para usos urbanos que no requieren de agua potable, reduciendo de esta forma tanto los volúmenes provenientes de la ITAM de la Tordera, como el gasto energético de la instalación. Concretamente, el agua regenerada se destina a la limpieza del espacio público, al riego de campos de golf, zonas agrícolas y jardines de propiedad municipal y a la recarga de acuíferos y aguas subterráneas.

\section{Figura 1}

\section{LOCALIZACIÓN DE LOS ESTABLECIMIENTOS HOTELEROS Y LAS PRINCIPALES ATRACCIONES TURÍSTICAS DE LLORET DE MAR}

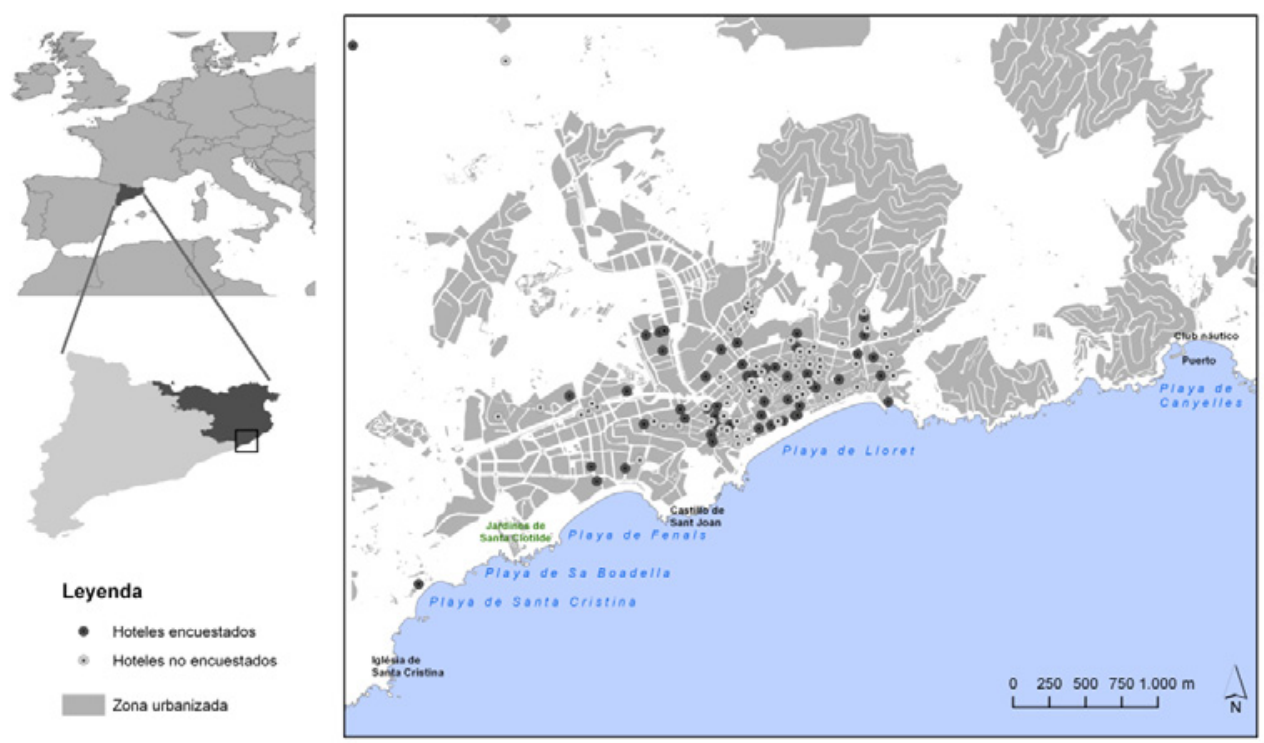

\section{METODOLOGÍA}

Lloret de Mar contaba durante el verano de 2014 con 124 establecimientos hoteleros. Para el presente estudio, de estos 124 establecimientos, se eliminaron 11 debido a causas diversas. Por ejemplo, algunos de ellos estuvieron cerrados durante todo el verano o se encontraban bajo una nueva gerencia en el momento de iniciar el trabajo de campo, lo que hacía imposible obtener los datos del año anterior. En otros casos, pese a que estaban considerados como 
establecimientos hoteleros independientes, a efectos prácticos formaban parte de otro, tanto a nivel del cómputo de pernoctaciones como de facturación del agua consumida. La población de estudio disponible fue, pues, de 113 establecimientos. Según el año de construcción de cada uno de los establecimientos y de su capacidad hotelera (en número de plazas), la representatividad de la muestra analizada es elevada especialmente en relación a la antigüedad de los hoteles (tabla 3). Sin embargo, referente a su capacidad, existen dos segmentos que no están bien representados. Los hoteles con menos de 100 plazas están infrarrepresentados (28,30\% de la muestra frente al 34,51 del total de la población disponible) y los de más de 700 plazas sobredimensionados (15,09\% de la muestra frente al 7,96 de la población).

\section{Tabla 3}

\section{CARACTERIZACIÓN DE LA POBLACIÓN DISPONIBLE Y LA MUESTRA SEGÚN LAS VARIABLES AÑO DE CONSTRUCCIÓN Y CAPACIDAD DE ALOJAMIENTO DE LOS HOTELES DE LLORET DE MAR}

\begin{tabular}{|c|c|c|c|c|}
\hline & \multicolumn{2}{|c|}{ Población disponible } & \multicolumn{2}{|c|}{ Muestra } \\
\hline & Núm. & $\%$ & Núm. & $\%$ \\
\hline \multicolumn{5}{|l|}{ Año de construcción } \\
\hline $1959 \mathrm{o}$ anterior & 15 & 13,27 & 8 & 15,09 \\
\hline 1960-1969 & 43 & 38,05 & 18 & 33,96 \\
\hline $1970-1979$ & 34 & 30,08 & 15 & 28,30 \\
\hline 1980-1989 & 11 & 9,73 & 4 & 7,54 \\
\hline 1990-1999 & 6 & 5,30 & 4 & 7,54 \\
\hline 2000 a la actualidad & 4 & 3,54 & 4 & 7,54 \\
\hline \multicolumn{5}{|c|}{ Capacidad de alojamiento } \\
\hline$<100$ plazas & 39 & 34,51 & 15 & 28,30 \\
\hline$>100-<200$ & 23 & 20,35 & 8 & 15,09 \\
\hline$>200-<300$ & 14 & 12,38 & 6 & 11,32 \\
\hline$>300-<400$ & 9 & 7,96 & 4 & 7,54 \\
\hline$>400-<500$ & 8 & 7,07 & 5 & 9,43 \\
\hline$>500-<600$ & 7 & 6,19 & 4 & 7,54 \\
\hline$>600-<700$ & 4 & 3,53 & 3 & 5,66 \\
\hline$>700$ plazas & 9 & 7,96 & 8 & 15,09 \\
\hline Total & 113 & 100 & 53 & 100 \\
\hline
\end{tabular}

Fuente: Elaboración propia a partir de los datos publicados en la Guía Oficial de Establecimientos Turísticos de Cataluña y en la Sede Electrónica del Catastro.

Entre los meses de junio y octubre de 2014, se llevaron a cabo 53 encuestas a los hoteles del municipio (45\% de la población). De acuerdo con la legislación catalana (DOGC, 2010), los establecimientos hoteleros se dividen en dos grandes grupos: los hoteles, establecimientos con una categorización en estrellas, y los hostales y pensiones, comparables a los hoteles pero sin un sistema de clasificación en estrellas. Para el presente trabajo, el término hotel incluye los hoteles, hostales y pensiones, excepto en los casos debidamente indicados. 
Las encuestas tenían una duración media de 30 minutos y se estructuraban en 3 apartados: (1) datos generales del establecimiento, (2) servicios e infraestructuras del establecimiento con requerimiento hídrico y (3) medidas de ahorro hídrico adoptadas y no adoptadas (ver variables exploradas en la tabla 4). En el apartado 3, referente a la adopción de medidas de ahorro, se pedía al encuestado que respondiera cuáles eran las medidas de ahorro hídrico implementadas, y cuáles no se tenía previsión de hacerlo a corto plazo. El abanico inicial de respuestas era de 17 posibles respuestas, a parte de otras no incluidas en la encuesta pero que el encuestado considerara oportuno añadir. De cada una de ellas, se pedía el motivo principal de la implementación o no de la medida en cuestión. En el primer caso el encuestado debía elegir uno de los siguientes motivos ya apuntados por Ayuso (2007): (1) reducción de costes, (2) preocupación por el medio ambiente, (3) respuesta a las demandas de los clientes, (4), porque la normativa me obliga, (5) mejora de la imagen del hotel y (6) mejora del márquetin y la publicidad. En el segundo caso, la no adopción de medidas podía ser debida a: (1) elevada inversión económica, (2) falta de tiempo y/o conocimiento, (3) pone en peligro la satisfacción del cliente, (4) dificultad para involucrar a los empleados, (5) el sector hotelero no tiene que adoptar medidas de ahorro hídrico y (7) desconozco como acceder a más información para poder ahorrar más agua.

La tabla 4 muestra la descripción estadística de las variables analizadas en el presente trabajo. A grandes rasgos, más del $65 \%$ de los hoteles encuestados son de categoría 3 y 4 estrellas, mientras que el grupo con menor representación lo forman los hoteles de 1 y 2 estrellas $(15,09 \%)$. La muestra representada comprende tanto hoteles que forman parte de una cadena $(49,06 \%)$ como hoteles de gestión independiente $(50,94 \%)$. Muchos de ellos, el 77,36\% fueron construidos antes de la década de 1980. Solo 5 de los hoteles encuestados $(9,43 \%)$ disponen de una o más certificaciones ambientales y/o de calidad. Además, la media de la capacidad hotelera de la muestra es de 355,9 plazas y la media de la superficie construida es de $8.037,76 \mathrm{~m}^{2}$.

Con los información resultante de los apartados 1, 2 y 3 de las encuestas, se ha realizado un análisis jerárquico de clústeres con el fin de determinar los grandes grupos de establecimientos con patrones similares en relación al nivel de implementación de las medidas de ahorro hídrico y el grado de sensibilización de los gestores. Para ello, las variables han sido convertidas en binarias cuando en aquellos casos que se ha considerado necesario. Por ejemplo, respecto al año de construcción del establecimiento, se ha creado una nueva variable, "Antigüedad", con la que se separan aquellos hoteles construidos antes de 1980 (momento a partir del cual Lloret de Mar y la Costa Brava en general entran en su fase de estancamiento) (Butler, 2006), de los que fueron construidos posteriormente. Referente a las medidas de ahorro hídrico, y con el fin de determinar si las razones que llevan al gestor a aplicarlas son distintas en función del nivel tecnológico y/o económico de cada una de ellas, se han diferenciado los motivos que explican la implementación o no de las medidas según si son básicas o avanzadas. Con todo ello, se han determinado 5 clústeres creados a partir del método Ward. El dendrograma resultante del análisis clúster mostraba que 5 era el número de grupos que mejor representaba la estructura de datos. Para este análisis, se ha utilizado el paquete RCommander del software estadístico $R$ (R Development Core Team, 2010). 
Tabla 4

DESCRIPCIÓN ESTADÍSTICA DE LA MUESTRA ANALIZADA SEGÚN LAS VARIABLES EXPLORADAS

\begin{tabular}{|c|c|c|c|}
\hline & & Frecuencia & Porcentaje (\%) \\
\hline \multicolumn{4}{|l|}{ (1) Datos generales } \\
\hline \multirow{6}{*}{ Categoría } & Hostales-pensiones & 6 & 11,32 \\
\hline & $*$ & 5 & 9,43 \\
\hline & *** & 3 & 5,66 \\
\hline & $* * *$ & 16 & 30,19 \\
\hline & $* * * *$ & 19 & 35,85 \\
\hline & ****** & 4 & 7,55 \\
\hline \multirow{2}{*}{ Construcción posterior al año 1980} & Sí & 12 & 22,64 \\
\hline & No & 41 & 77,36 \\
\hline \multirow{2}{*}{ Afiliación cadena hotelera } & Sí & 26 & 49,06 \\
\hline & No & 27 & 50,94 \\
\hline \multirow{2}{*}{ Certificaciones ambientales y/o de calidad } & Sí & 5 & 9,43 \\
\hline & No & 48 & 90,57 \\
\hline \multicolumn{4}{|c|}{ (2) Servicios e infraestructuras con requerimiento hídrico } \\
\hline \multirow{2}{*}{ Jardines y zonas verdes } & Sí & 17 & 32,08 \\
\hline & No & 36 & 67,92 \\
\hline \multirow{2}{*}{ Piscina } & Sí & 43 & 81,13 \\
\hline & No & 10 & 18,87 \\
\hline \multirow{2}{*}{ Spa } & Sí & 21 & 39,62 \\
\hline & No & 32 & 60,38 \\
\hline \multirow{2}{*}{ Lavandería } & Sí & 32 & 60,38 \\
\hline & No & 21 & 39,62 \\
\hline \multirow{2}{*}{ Servicio de cocina } & Sí & 47 & 88,68 \\
\hline & No & 6 & 11,32 \\
\hline \multicolumn{4}{|l|}{ (3) Medidas de ahorro hídrico } \\
\hline \multirow{3}{*}{ Medidas de ahorro implementadas } & Ninguna & 3 & 5,66 \\
\hline & Entre 1 y 3 & 30 & 56,60 \\
\hline & Más de 3 & 20 & 37,74 \\
\hline
\end{tabular}

\section{RESULTADOS}

Los resultados del análisis de clústeres se muestran en la tabla 5. Cada uno de ellos contiene como mínimo el $11,32 \%$ de la muestra analizada, con lo que se mantiene una representatividad proporcional en cada uno de los grupos creados. Con todas las variables exploradas se pretende ilustrar, entre otros elementos, una aproximación al nivel de proactividad, o cuán responsable es el hotel en lo que se refiere al ahorro de agua; de sensibilización ambiental, o en qué grado el gestor hotelero muestra una preocupación por el medio ambiente; y de tecnificación, en función de si el hotel dispone o no de un nivel alto de tecnología aplicada a reducir el consumo hídrico. 


\section{Tabla 5}

\section{CLÚSTERES CREADOS A PARTIR DE LAS VARIABLES EXPLORADAS}

\begin{tabular}{|c|c|c|c|c|c|}
\hline Variable & $\begin{array}{l}\text { Grupo A } \\
(30,18 \%)\end{array}$ & $\begin{array}{l}\text { Grupo B } \\
(20,75 \%)\end{array}$ & $\begin{array}{l}\text { Grupo C } \\
(20,75 \%)\end{array}$ & $\begin{array}{l}\text { Grupo D } \\
(16,98 \%)\end{array}$ & $\begin{array}{l}\text { Grupo E } \\
(11,32 \%)\end{array}$ \\
\hline \multicolumn{6}{|l|}{ Categoría } \\
\hline Hostales - pensiones & 0 & 0 & 0 & 0 & $\odot \odot \square \odot \odot$ \\
\hline Categoría media-baja (1, 2 y 3 estrellas) & $\odot \odot$ & $\odot \odot \odot \odot$ & 0 & $\odot \odot \odot \odot$ & 0 \\
\hline Categoría alta (4 y 5 estrellas) & $\odot \odot \odot \odot$ & $\odot$ & $\odot \odot-\odot \odot$ & $\odot$ & 0 \\
\hline \multicolumn{6}{|l|}{ Dimensión del establecimiento } \\
\hline Más de 400 plazas & $\odot \odot \odot$ & $\odot$ & $\odot \odot \odot$ & $\odot \odot \odot$ & 0 \\
\hline Más de $5.000 \mathrm{~m}^{2}$ & $\odot \odot \bullet \odot$ & $\odot$ & $\odot \odot \odot \odot$ & $\odot \odot \odot$ & 0 \\
\hline \multicolumn{6}{|l|}{ Propiedad y gestión medioambiental } \\
\hline El hotel funciona desde el año 1980 o posterior & $\odot$ & $\odot$ & $\odot \odot \odot \odot$ & 0 & $\odot \odot \odot$ \\
\hline El hotel forma parte de una cadena hotelera & $\odot \odot \odot$ & 0 & $\odot \odot \bullet \odot$ & $\odot \odot \bullet \odot$ & 0 \\
\hline Hotel con certificación de calidad y/o ambiental & $\odot \odot$ & 0 & $\odot$ & 0 & 0 \\
\hline \multicolumn{6}{|l|}{ Servicios } \\
\hline Jardín y zonas verdes & $\odot \odot \odot \odot$ & 0 & $\odot \odot \odot$ & $\odot$ & $\odot$ \\
\hline Piscina & $\odot \odot \square \odot \odot$ & $\odot \odot \odot \odot$ & $\odot \odot \odot \odot$ & 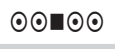 & $\odot \odot \odot \odot$ \\
\hline Spa & $\odot \odot \bullet \odot$ & $\odot \odot$ & $\odot \odot \odot$ & 0 & 0 \\
\hline Lavandería & $\odot \odot \odot \odot$ & $\odot \odot \bullet \odot \odot$ & $\odot \odot \odot$ & $\odot$ & $\odot \odot \mathbf{r} \odot$ \\
\hline Cocina y comedor & $\odot \odot \bullet \odot$ & $\odot \odot \odot \odot$ & $\odot \odot \bullet \odot \odot$ & $\odot \odot \bullet \odot$ & 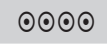 \\
\hline \multicolumn{6}{|l|}{ Medidas de ahorro implementadas: } \\
\hline Ninguna & 0 & 0 & 0 & 0 & $\odot \odot \odot$ \\
\hline Entre 1 y 3 & 0 & $\odot \odot \mathbf{r} \odot$ & $\odot \odot \mathbf{r} \odot$ & $\odot \odot \mathbf{r} \odot$ & $\odot$ \\
\hline Más de 3 & $\odot \odot \odot \odot$ & $\odot$ & 0 & 0 & $\odot \odot$ \\
\hline \multicolumn{6}{|c|}{ Principales incentivos que explican la implementación de medidas de ahorro: } \\
\hline \multicolumn{6}{|c|}{ FACTORES LEGALES Y/O NORMATIVOS } \\
\hline \multicolumn{6}{|l|}{ Porque estoy obligado a cumplir con las normativas, } \\
\hline he implementado 10 más iniciativas básicas & $\odot \odot$ & $\odot$ & 0 & 0 & 0 \\
\hline $\begin{array}{l}\text { he implementado } 1 \text { o más iniciativas } \\
\text { avanzadas }\end{array}$ & 0 & 0 & 0 & 0 & 0 \\
\hline \multicolumn{6}{|l|}{ FACTORES SOCIALES } \\
\hline \multicolumn{6}{|c|}{ Porque estoy preocupado por el medioambiente y procuro ser sostenible, } \\
\hline he implementado 10 más iniciativas básicas & $\odot \odot \odot$ & $\odot \odot$ & $\odot$ & 0 & $\odot$ \\
\hline $\begin{array}{l}\text { he implementado } 1 \text { o más iniciativas } \\
\text { avanzadas }\end{array}$ & $\odot$ & 0 & 0 & 0 & 0 \\
\hline \multicolumn{6}{|l|}{ FACTORES ECONÓMICOS } \\
\hline \multicolumn{6}{|l|}{ Para reducir los costes asociados al consumo de agua, } \\
\hline he implementado 10 más iniciativas básicas & $\odot \odot \mathbf{\odot \odot ~}$ & $\odot \odot \mathbf{\odot \odot ~}$ & $\odot \odot \mathbf{\odot} \odot$ & $\odot \odot \mathbf{\odot \odot ~}$ & $\odot \odot$ \\
\hline $\begin{array}{l}\text { he implementado } 1 \text { o más iniciativas } \\
\text { avanzadas }\end{array}$ & $\odot \odot$ & 0 & $\odot \odot$ & 0 & 0 \\
\hline
\end{tabular}




\section{FACTORES ESTRATIÉGICOS Y/ EMIPRESARIALES}

Porque los clientes así lo piden,

$\begin{array}{lllllll}\text { he implementado } 1 \text { o más iniciativas básicas } & 0 & 0 & 0 & 0 & 0 \\ \begin{array}{l}\text { he implementado } \\ \text { avanzadas }\end{array} & \begin{array}{l}0 \\ \text { a más iniciativas }\end{array} & 0 & 0 & 0 & 0 & 0\end{array}$

Porque quiero mejorar la imagen del hotel,

$\begin{array}{llllllll}\text { he implementado } 10 \text { más iniciativas básicas } & 0 & 0 & 0 & 0 & 0 \\ \begin{array}{l}\text { he implementado } \\ \text { avanzadas }\end{array} & 0 \text { más iniciativas } & 0 & 0 & 0 & 0 & 0\end{array}$
avanzadas

Porque quiero mejorar la publicidad y el marketing del hotel, he implementado 10 más iniciativas básicas

$0 \quad 0$

he implementado 1 o más iniciativas

0

0

0

0 avanzadas

0

0

0

Principales barreras que explican el rechazo a llevar a cabo medidas de ahorro:

FACTORES LEGALES Y/O NORMATIVOS

Porque no tengo acceso a más información sobre cómo puedo ahorrar aún más agua,

\begin{tabular}{|c|c|c|c|c|c|}
\hline $\begin{array}{l}\text { no voy a implementar ninguna iniciativa } \\
\text { básica }\end{array}$ & $\odot \odot \bullet \odot$ & $\odot \odot \bullet \odot$ & $\odot \odot \bullet^{\circ}$ & $\odot \odot \bullet \odot$ & $\odot \odot \boldsymbol{\bullet \odot ~}$ \\
\hline $\begin{array}{l}\text { no voy a implementar ninguna iniciativa } \\
\text { avanzada }\end{array}$ & $\odot \odot \bullet \odot \odot$ & $\odot \odot \bullet \odot \odot$ & $\odot \odot \bullet \odot \odot$ & $\odot \odot \bullet \odot$ & $\odot \odot \boldsymbol{\bullet \odot ~}$ \\
\hline \multicolumn{6}{|l|}{ FACTORES SOCIALES } \\
\hline \multicolumn{6}{|l|}{ Debido a la falta de tiempo y/o conocimiento, } \\
\hline $\begin{array}{l}\text { no voy a implementar ninguna iniciativa } \\
\text { básica }\end{array}$ & $\odot$ & $\odot \odot$ & $\odot \odot \odot$ & 0 & $\odot$ \\
\hline $\begin{array}{l}\text { no voy a implementar ninguna iniciativa } \\
\text { avanzada }\end{array}$ & $\odot \odot$ & $\odot \odot$ & $\odot$ & 0 & 0 \\
\hline
\end{tabular}

Porque el sector hotelero no tiene que ahorrar agua,

\begin{tabular}{|c|c|c|c|c|c|}
\hline $\begin{array}{l}\text { no voy a implementar ninguna iniciativa } \\
\text { básica }\end{array}$ & 0 & 0 & 0 & 0 & 0 \\
\hline $\begin{array}{l}\text { no voy a implementar ninguna iniciativa } \\
\text { avanzada }\end{array}$ & 0 & 0 & 0 & 0 & 0 \\
\hline \multicolumn{6}{|l|}{ FACTORES ECONÓMICOS } \\
\hline \multicolumn{6}{|l|}{ Debido a la elevada inversión económica, } \\
\hline $\begin{array}{l}\text { no voy a implementar ninguna iniciativa } \\
\text { básica }\end{array}$ & $\odot$ & $\odot \odot \odot \odot$ & $\odot \odot$ & $\odot$ & 0 \\
\hline $\begin{array}{l}\text { no voy a implementar ninguna iniciativa } \\
\text { avanzada }\end{array}$ & $\odot \odot$ & $\odot \odot \bullet \odot$ & $\odot \odot \bullet \odot \odot$ & $\odot \odot$ & 0 \\
\hline \multicolumn{6}{|l|}{ FACTORES ESTRATÉGICOS Y/ EMPRESARIALES } \\
\hline \multicolumn{6}{|l|}{ Porque pone en peligro la satisfacción del cliente, } \\
\hline $\begin{array}{l}\text { no voy a implementar ninguna iniciativa } \\
\text { básica }\end{array}$ & $\odot \odot$ & 0 & 0 & 0 & 0 \\
\hline $\begin{array}{l}\text { no voy a implementar ninguna iniciativa } \\
\text { avanzada }\end{array}$ & $\odot \odot \odot$ & $\odot \odot$ & $\odot \odot \odot \odot$ & $\odot \odot \odot$ & $\odot$ \\
\hline \multicolumn{6}{|l|}{ Porque tengo dificultades para involucrar al personal empleado, } \\
\hline $\begin{array}{l}\text { no voy a implementar ninguna iniciativa } \\
\text { básica }\end{array}$ & 0 & 0 & 0 & 0 & 0 \\
\hline $\begin{array}{l}\text { no voy a implementar ninguna iniciativa } \\
\text { avanzada }\end{array}$ & 0 & 0 & 0 & 0 & 0 \\
\hline
\end{tabular}

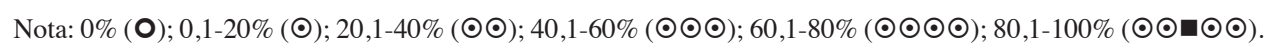

Cuadernos de Turismo, 41, (2018), 187-218 


\subsection{Grupo A. Hoteles antiguos con gran capacidad de alojamiento, preocupados por el medioambiente $\mathbf{y}$ altamente tecnificados}

Formado por el 30,18\% de la muestra, integrado mayoritariamente por establecimientos hoteleros de alta categoría (62\%) aunque también encontramos hoteles de categoría media y/o baja (38\%). Son establecimientos con una gran capacidad de alojamiento, tanto en número de plazas como en superficie construida. Ocho de ellos disponen de más de 400 plazas, incluyendo el hotel con mayor capacidad de todos los encuestados (1.740 plazas). La mayor parte de ellos fueron construidos antes de 1980. Concretamente, ocho se construyeron durante la década de los sesenta o anterior y siete durante la década de los setenta. Solo uno de ellos fue construido después del año 2000. Se caracterizan por disponer de prácticamente todos aquellos servicios hoteleros con mayores requerimientos hídricos (jardín, piscina, servicios termales, lavandería y cocina). Es importante destacar que es el grupo que cuenta con un mayor número de hoteles con algún tipo de certificación tanto ambiental como de calidad (el 25\% de establecimientos de este grupo disponen de certificación). En consonancia con este factor, es también el grupo que más medidas de ahorro de agua ha llevado a cabo (todos los hoteles de este grupo han implementado 3 o más medidas). Es también el grupo que presenta mayor proporción de medidas avanzadas aplicadas $(31,25 \%)$. Los principales incentivos que explican la elevada implementación de medidas de ahorro, incluyendo tanto básicas como avanzadas, están básicamente relacionadas con la reducción de los costes derivados del consumo de agua $(81,25 \%)$, la preocupación medioambiental de los gestores hoteleros $(50 \%)$ y la obligatoriedad que imponen las normativas y la legislación competente (25\%). En este grupo, existe una única razón que explica el rechazo a llevar a cabo más medidas de ahorro ya sean básicas o avanzadas: el gestor hotelero desconoce cómo se puede reducir más el consumo de agua.

\subsection{Grupo B. Pequeños hoteles antiguos preocupados por el medioambiente pero poco tecnificados}

Este grupo está formado por el 20,75\% de la muestra y está integrado prácticamente en su totalidad por hoteles de media y/o baja categoría. Son hoteles pequeños, todos ellos con una capacidad de alojamiento inferior a 400 plazas, y de propiedad independiente. Cabe destacar que es el grupo con mayor número de hoteles de menos de 100 plazas. Respecto a su antigüiedad, todos excepto en un caso fueron construidos antes de 1980. Concretamente, ocho de ellos presentan una planta construida en la década de los sesenta o anterior y tres fueron construidos durante las décadas de los setenta y ochenta. Se caracterizan por disponer de distintos servicios con requerimientos hídricos, excepto jardín y zonas verdes. Muestran un nivel de proactividad medio ya que el $81 \%$ de los hoteles de este grupo han implementado entre 1 y 3 medidas de ahorro. Es un grupo que presenta un nivel de preocupación ambiental medio. Así, el 27\% de los hoteles de este grupo ha desarrollado medidas de ahorro hídrico básicas debido a que están preocupados por el medioambiente y procuran ser sostenibles. A pesar de ello, muestran un nivel tecnológico bajo puesto que en ningún caso han aplicado medidas avanzadas. Referente a las barreras, destaca la oposición que muestran a llevar a cabo nuevas medidas de ahorro, ya sean básicas o avanzadas, argu- 
mentando la elevada inversión económica inicial que requieren (el 90\% de este grupo no desarrollará ninguna medida avanzada y el $63 \%$ ninguna medida básica). Además, existe también un componente de desconocimiento relativo a cómo ahorrar más agua (100\%).

\subsection{Grupo C. Nuevos hoteles afiliados a cadenas hoteleras, altamente tecnificados y medianamente proactivos}

Este grupo reúne el $20,75 \%$ de los hoteles encuestados, todos ellos de categoría alta y con una elevada capacidad de alojamiento (el 54\% dispone de 400 o más plazas de alojamiento). Es el grupo con mayor número de hoteles construidos después de 1980 (63\%): dos de ellos en la década de los ochenta, tres en la de los noventa y dos posteriormente al año 2000. Todos los individuos de este grupo forman parte de una cadena hotelera. Tienen un nivel medio de proactividad, puesto que todos ellos han implementado entre 1 y 3 medidas de ahorro. Aunque solo el $9 \%$ de los hoteles disponen de algún tipo de certificación, hay que destacar que es el segundo grupo en número de hoteles certificados. En cuanto a los principales incentivos que perciben cuando se trata de llevar a cabo medidas de ahorro hídrico, todos los encuestados destacan la reducción de costes económicos relacionados con el consumo de agua y, aunque en mucha menor medida, la preocupación por el medioambiente $(9,09 \%)$. Igual que el A, este grupo muestra también un nivel destacado de tecnificación ya que el $27 \%$ de los hoteles han instalado medidas avanzadas destinadas a conseguir un mayor ahorro hídrico. En cuanto a los motivos según los cuales los gestores rechazan la aplicación de otras medidas, destaca la amplia variabilidad en las respuestas. El desconocimiento relativo a cómo ahorrar más agua es una de las principales barreras en todos los casos de este grupo, como se ha apreciado en el resto de grupos. La elevada inversión económica $(81,81 \%)$ y la falta de tiempo $(54,54 \%)$ son otras de las principales barreras que explican el rechazo a llevar a cabo más medidas de ahorro ya sean básicas y/o avanzadas. Es importante destacar que es el grupo con mayor número de hoteles que rechazan la implementación de medidas de ahorro en base al argumento de que puede poner en peligro la satisfacción del cliente $(72,72 \%)$.

\subsection{Grupo D. Hoteles antiguos afiliados a cadenas hoteleras, poco preocupados por el medio ambiente y poco tecnificados}

Grupo que representa el $16,98 \%$ de la muestra encuestada y formado principalmente por hoteles de media y baja categoría. Son hoteles con alta capacidad de alojamiento (el $55 \%$ dispone de más de 400 plazas) y es el único grupo que no reúne a ningún hotel con menos de 100 plazas. Es el grupo que reúne los hoteles más antiguos del municipio puesto que ninguno de ellos fue construido después de 1980: 5 hoteles se construyeron durante la década de los 60 y 4 en la de los setenta. A pesar de esto, el $88 \%$ de los establecimientos de este grupo pertenecen a una cadena hotelera. Es el grupo que dispone de una menor variedad de servicios con requerimientos hídricos: ninguno de ellos ofrece servicios de spa y solo el $11 \%$ dispone de lavandería y de zonas verdes. Igual que el grupo anterior, los hoteles del grupo D presentan un nivel de proactividad medio ya que todos ellos tienen entre 1 y 3 medidas de ahorro hídrico desarrolladas. Es un grupo poco tecnificado puesto 
que en ningún caso han instalado medidas avanzadas. A pesar de ello, todos los hoteles del grupo han llevado a cabo alguna medida básica debido a la única voluntad de reducir los costes económicos relacionados con el consumo de agua. Respecto a las barreras percibidas por los gestores, y en la misma línea que el resto de grupos, todos los hoteles de este grupo manifiestan no tener acceso a información sobre cómo ahorrar más agua. Por último, destacar que el $44 \%$ de los hoteles expresan que no van a instalar medidas avanzadas por miedo a poner en peligro la comodidad y confort del cliente.

\subsection{Grupo E. Pequeños hostales y pensiones independientes, de nueva construcción, poco tecnificados y con un nivel de proactividad bajo}

Este último grupo reúne al 11,32\% de los establecimientos hoteleros de la muestra. Todos los hoteles de este grupo son hostales o pensiones con baja capacidad de alojamiento (todos excepto uno disponen de menos de 100 plazas) y con menor superficie construida. La mitad de ellos son establecimientos construidos después de 1980, aunque la variable antigüedad presenta una elevada variabilidad: dos de los hoteles de este grupo fueron construidos durante la década de los sesenta y los cuatro restantes en los años 70s, 80s, 90 s y 2000 respectivamente. Disponen de todos los servicios hoteleros con mayor requerimiento hídrico, a excepción de jardines y spa. Es el grupo con el nivel de proactividad más bajo: la mitad de ellos no han desarrollado ninguna medida de ahorro hídrico aunque presentan un nivel medio de sensibilización medioambiental (el 16\% han implementado alguna medida básica por motivos relacionados con la sostenibilidad ambiental). Otro de los incentivos más destacados es el relacionado con la reducción de costes en las facturas del agua. De este modo, el 33\% de los hoteles de este grupo han llevado a cabo medidas básicas con la intención de reducir los costes económicos relacionados con el consumo. Respecto al rechazo a implementar otras medidas de ahorro, la barrera más detectada en todos los hoteles de este grupo es, como en el resto de grupos, el desconocimiento en cómo ahorrar más agua o a qué tecnologías recurrir para reducir el consumo.

\section{DISCUSIÓN}

A partir de un análisis de clústeres de los establecimientos hoteleros de una destinación turística internacional como es Lloret de Mar se han podido explorar las variables relacionadas con el número de medidas de ahorro hídrico aplicadas y los principales incentivos y barreras existentes para su implementación.

La tabla 6 sintetiza los incentivos y las barreras principales que explican la aplicación o no de medidas de ahorro hídrico a partir de las cinco grandes tipologías hoteleras en función de sus niveles de proactividad, sensibilización y tecnificación. Las medidas más populares y que aparecen en todos los grupos corresponden a las básicas. Como apuntábamos al inicio de este estudio, son iniciativas que o bien son de obligatoria instalación debido a las normativas ambientales y/o urbanísticas o que requieren de una clara intencionalidad de implementación por parte de los gestores pero no suponen ni una gran inversión económica ni un elevado nivel de conocimientos técnicos. Las cuatro medidas más desarrolladas son la sensibilización del personal (43 establecimientos), los 
aireadores en los grifos (36), la sensibilización al cliente (34) y la doble pulsación en los sanitarios (31). En el otro extremo, las medidas avanzadas, las que requieren de una notable inversión económica y precisan de un conocimiento técnico avanzado, son las que presentan un menor número de casos en los grupos A y C $(31,25$ y 27,27\% respectivamente) siendo cero en el resto de grupos. Las medidas que, por ejemplo, inciden en el tratamiento y la reutilización de las aguas grises o pluviales implican, además, una elevada capacidad para innovar desde el punto de vista tecnológico (Kasim et al., 2014). En este punto es interesante incorporar el ejemplo de un caso paradigmático de ahorro hídrico en el sector hotelero de Lloret de Mar: el hotel Samba. Este hotel de 3 estrellas y con más de 800 plazas fue el primer hotel en Europa en obtener dos certificaciones ambientales casi de forma consecutiva: la ISO14001 en 1998 y el EMAS al año siguiente. Este hotel desarrolló y implementó un sistema de recuperación de aguas grises de las duchas de las habitaciones para ser reutilizadas en las cisternas de los sanitarios gracias al cual el ahorro anual acumulado desde 1997 hasta 2013 asciende a más de $160.000 \mathrm{~m}^{3}$ de agua. Para el mismo período, el consumo por pernoctación ha disminuido un $80 \%$ (de 372 litros por pernoctación en 1997 a 77,5 en 2013) (Gabarda-Mallorquí, Garcia y Ribas, 2017). No cabe la menor duda que el éxito obtenido para el caso del hotel Samba en la aplicación de este tipo de medidas avanzadas rompe una lanza a favor de la introducción de este tipo de medidas en el sector hotelero.

\section{Tabla 6}

\section{CARACTERÍSTICAS PRINCIPALES Y NIVELES DE PROACTIVIDAD, SENSIBILIZACIÓN Y TECNIFICACIÓN EN RELACIÓN AL AHORRO HÍDRICO DE CADA UNA DE LAS TIPOLOGÍAS HOTELERAS.}

\begin{tabular}{cccccccc}
\hline & $\begin{array}{c}\text { Categoría } \\
\text { alta }\end{array}$ & Maduro & $\begin{array}{c}\text { Alta } \\
\text { capacidad } \\
\text { alojamiento }\end{array}$ & $\begin{array}{c}\text { Hotel } \\
\text { afiliado a } \\
\text { cadena }\end{array}$ & Proactivo & Sensibilizado & $\begin{array}{c}\text { Altamente } \\
\text { tecnificado }\end{array}$ \\
\hline A & $\checkmark$ & $\checkmark$ & $\checkmark$ & - & $\checkmark$ & $\checkmark$ & $\checkmark$ \\
\hline B & $x$ & $\checkmark$ & $x$ & $x$ & - & $\checkmark$ & $x$ \\
\hline C & $\checkmark$ & $x$ & $\checkmark$ & $\checkmark$ & - & - & $\checkmark$ \\
\hline D & $x$ & $\checkmark$ & - & $\checkmark$ & - & $x$ & $x$ \\
\hline E & $x$ & $x$ & $x$ & $x$ & $x$ & - & $x$ \\
\hline
\end{tabular}

El mayor o menor número de medidas de ahorro hídrico implementadas a menudo guarda una estrecha relación con el tamaño del hotel, tanto en número de plazas como en superficie construida (Kasim et al., 2014). En el presente estudio, el grupo de establecimientos con más medidas aplicadas (más de 3) lo forman un conjunto heterogéneo de hoteles de categorías alta, media y baja con más de 400 plazas y más de $5.000 \mathrm{~m}^{2}$ construidos que ofrecen una amplia variedad de servicios con requerimiento hídrico como piscinas, espacios verdes o lavandería, entre otros (grupo A). Cabe destacar que es también el grupo con un mayor número de hoteles con certificados ambientales y/o de calidad. 
Además, éste es el grupo que se muestra más preocupado por el medio ambiente, ya que una de las motivaciones principales que explican la implementación de medidas, ya sean básicas o avanzadas, está relacionada con "Estoy preocupado por el medio ambiente y procuro ser sostenible". A pesar de esto, en el otro extremo, los establecimientos sin ninguna medida aplicada lo forman mayoritariamente pequeños hostales y pensiones (grupo E). En este sentido, en Lloret de Mar sucede lo mismo que constatan otros estudios según los cuales los establecimientos hoteleros de reducidas dimensiones y con un menor número de equipamientos, servicios y espacios con mayor requerimiento hídrico generan un impacto medioambiental menor (Videras \& Alberini, 2000), razón que podría explicar el bajo nivel de proactividad mostrado en este grupo. No obstante, también se debe tener presente que tienen menor capacidad económica y/o técnica para minimizarlo a partir de, por ejemplo, la aplicación de medidas de ahorro de agua (Kasim et al., 2014). La afiliación a una cadena hotelera merece una pequeña mención en este punto puesto que, según los resultados obtenidos en este caso de estudio, es un factor que no parece decisivo ni en el nivel de proactividad ni en el de preocupación medioambiental ni en el de tecnificación.

A nivel general, la motivación principal que explica la implementación de medidas de ahorro hídrico y que se intuye en todos los grupos está relacionada con la reducción de costes económicos asociados a las facturas de agua, como sucede en otros casos de estudio (Ayuso, 2007; Chan \& Hawkins, 2010). Aunque la preocupación por el medio ambiente es la segunda motivación que explica la adopción de medidas de ahorro por parte de los hoteles encuestados, su representatividad es muy baja comparada con la del incentivo asociado a la reducción de costes. Por otra parte, se ha demostrado que las grandes empresas emprenden buenas prácticas ambientales movidas por una mejora de su imagen y de su competitividad en el mercado (DiPietro, Cao, \& Partlow, 2013; Kasim et al., 2014; Nyahunzvi, 2013). Sin embargo, para el caso de Lloret de Mar, cabe destacar que ninguna de las medidas ha sido adoptada por razones de márquetin y mejora de la imagen del establecimiento ni a petición de los clientes. Probablemente habría que avanzar en esta línea y estudiar con mayor detalle cuáles son los efectos de una mejor publicidad de las medidas de ahorro hídrico implementadas en los establecimientos hoteleros respecto el número de clientes recibidos o de la valoración que éstos hacen de su estancia.

Referente a las principales barreras percibidas por el sector hotelero de Lloret de Mar, destaca que muchos de los encuestados desconocen o no tienen acceso a los medios necesarios para informarse sobre cómo ahorrar más agua. Este resultado coincide con otros estudios que destacan el poco o nulo acceso de los hoteles a este tipo de información (Ayuso, 2007; Chan, 2008). El segundo motivo que explica el rechazo a llevar a cabo medidas de ahorro, ya sean básicas y/o avanzadas, tiene que ver con la gran inversión económica que supone la instalación de dispositivos y/o mecanismos ahorradores de agua, especialmente cuando se trata de medidas avanzadas, o bien a los procesos de renovación de los certificados ambientales y/o de calidad, tal y como sucede en otros casos de estudio (Bramwell \& Alletorp, 2001; Stabler \& Goodall, 1997; Vernon et al., 2003). Cabe destacar que ninguno de los hoteles encuestados cree que el sector hotelero no tiene que realizar acciones para ahorrar agua y que por esto no han aplicado ninguna medida de ahorro, con lo que se intuye que el sector reconoce el impacto ambiental del turismo y es consciente que hay que proteger los recursos naturales como el agua. De ahí 
se deduce que el sector hotelero de Lloret de Mar se considera un actor estratégico en el momento de reducir los impactos derivados del consumo y sobreexplotación de recursos hídricos por parte del turismo, aunque a menudo, durante un proceso de encuestas, los individuos participantes aparentan estar preocupados por el medio ambiente porque se sienten presionados a mostrarse socialmente responsables y a tener un compromiso por la sostenibilidad medioambiental (Bohdanowicz, 2005). En futuras investigaciones sería importante aunar en esta línea.

\section{CONCLUSIONES}

El avance en el conocimiento de los incentivos y las barreras percibidas por los gestores hoteleros en el momento de implementar medidas de ahorro de agua es sin duda de vital importancia para la mejora en la sostenibilidad de los recursos hídricos en las destinaciones turísticas del litoral mediterráneo. En este artículo, a través de un análisis de clústeres, se han podido identificar y analizar las diversas tipologías de hotel que se dan actualmente en Lloret de Mar, una destinación turística receptora de importantes flujos turísticos internacionales y con una consolidada planta hotelera que concentra buena parte de la oferta de la Costa Brava.

El nivel de desarrollo de medidas de ahorro hídrico varía considerablemente en función de las características estructurales y organizativas de los hoteles que conforman cada uno de los grupos. Los establecimientos más proactivos y que muestran un mayor número de medidas de ahorro aplicadas, tanto básicas como avanzadas, se caracterizan por ser de categoría alta y por disponer de más superficie construida. Por ello, son también los que ofrecen buena parte de los servicios hoteleros que requieren más agua, tales como piscinas, zonas verdes o lavandería, entre otros. Así, y como era de suponer, la presencia de múltiples servicios e infraestructuras que requieren de agua para su funcionamiento se erige como factor clave para que los propios gestores hoteleros lleven a cabo medidas de ahorro para reducir el consumo de agua. Como se ha podido constatar, hay que destacar que aunque Lloret de Mar fue una de las primeras destinaciones turísticas internacionales del litoral español y que el desarrollo de su planta hotelera tuvo lugar a partir de mediados del siglo pasado, nos encontramos delante de un buen ejemplo de modernización y tecnificación de sus instalaciones y sus servicios hoteleros. Así, la antigüedad del establecimiento no minimiza el nivel de proactividad en relación al ahorro hídrico. De hecho, los hoteles más proactivos y más tecnificados forman parte de los primeros establecimientos que se abrieron al público antes de la década de los ochenta pero que adoptaron un proceso de modernización de sus instalaciones que revirtió también en una mayor eficiencia en el consumo de agua. Gracias a la presencia de estos hoteles altamente tecnificados y con certificados ambientales y/o de calidad, la transferencia de conocimientos entre establecimientos y la cooperación a través de órganos asociativos hoteleros debería convertirse en una valiosa herramienta para avanzar en una cada vez mayor eficiencia hídrica. Concretamente, hay que destacar el papel impulsor que podría asumir el Gremio Hotelero de Lloret de Mar, cuya consolidada estructura podría hacer posible la transferencia de conocimiento en la implementación de buenas prácticas de ahorro hídrico entre sus afiliados. 
La exploración y análisis de los principales incentivos cuando se trata de llevar a cabo medidas de ahorro permite llegar a las siguientes conclusiones. Por un lado, el incentivo más popular a la hora de emprender acciones para el ahorro hídrico es el relacionado con la reducción de los costes en las facturas de agua. Para ello, muchos de los hoteles encuestados han aplicado ciertas medidas con las que consiguen un menor consumo de agua y, en consecuencia, una reducción de costes. En este punto hay que destacar la elevada eficiencia de las medidas de ahorro hídrico que en el presente trabajo se han considerado básicas, ya que permiten un significativo ahorro económico pero que no precisan de una elevada inversión de instalación ni altos requerimientos técnicos. Por otro lado, existen establecimientos hoteleros altamente motivados por mejorar la calidad del medio ambiente y por ello tienen instalados distintos mecanismos avanzados que les permiten ahorrar agua. Los dos grupos que muestran un mayor nivel de preocupación y sensibilización ambiental son, como se ha podido apreciar, muy dispares entre ellos. Uno está formado por hoteles altamente proactivos, de categoría alta, altamente tecnificados y de grandes dimensiones. El otro lo componen establecimientos de categoría media-baja, poco tecnificados y de pequeñas dimensiones. Este hecho indica que una mayor predisposición y sensibilidad a la protección del medio ambiente es necesaria pero no suficiente para desarrollar, en este caso, medidas de ahorro hídrico. Aunque la consciencia y preocupación medioambiental sea un importante punto de partida, son necesarios otros factores para reducir el consumo de agua, como disponer de recursos económicos y/o tener acceso al conocimiento y la técnica necesarios.

En cuanto a las barreras percibidas, la inversión económica necesaria para la implementación de medidas de ahorro y el desconocimiento sobre cómo ahorrar más agua debido a un limitado acceso a la información necesaria, son dos de las barreras principales detectadas en este trabajo. De este modo, la administración, ya sea local, regional o nacional, debería jugar un papel más importante en el desarrollo y la implementación de medidas de ahorro hídrico en este sector hotelero. A través de programas informativos sobre el amplio abanico de posibilidades que pueden materializarse para ahorrar agua, subvenciones y ayudas o incentivos fiscales para aquellos hoteles que emprendan buenas prácticas se podrían superar algunas de las barreras percibidas por el sector hotelero. Con éstas y otras acciones, los gestores hoteleros podrían verse mejor asesorados para la adaptación de sus instalaciones y conseguir reducir el consumo de agua. Además, en el presente caso de estudio se intuye el grado de compromiso del sector hotelero frente a la minimización de los impactos ambientales derivados de las actividades turísticas. Así, ninguno de los hoteles encuestados ha negado el relevante papel que debe adoptar el propio sector hotelero en el momento de aplicar medidas de ahorro de agua en pro de la sostenibilidad de los recursos hídricos, aspecto éste que constituye un primer paso clave para avanzar en la reducción del consumo de agua y la consiguiente minimización de la presión sobre los recursos hídricos en destinaciones turísticas que, como es el caso de Lloret de Mar, el turismo se erige como el principal consumidor de agua.

La definición de cinco tipologías hoteleras en función de las medidas de ahorro hídrico desarrolladas, ha de ayudar a conocer de antemano los niveles de sensibilización, proactividad y tecnificación de los hoteles no incluidos en la muestra analizada en este estudio e incluso de futuros establecimientos hoteleros que se proyecten construir. Gracias a ello 
las administraciones locales y regionales podrán definir con más precisión las mejores estrategias de gestión de los recursos hídricos y del ahorro de agua en el sector turístico necesarias en caso de, por ejemplo, una supuesta ampliación de la oferta hotelera municipal. También en el caso de los hoteles con un nivel de sensibilización más bajo hacia el ahorro hídrico, sería interesante que las administraciones locales fomentaran acciones de concienciación ambiental dirigidas especialmente a este grupo de hoteles. Los establecimientos que presentan un nivel bajo de proactividad también deberían ser objeto de acciones de capacitación y empoderamiento promovidas desde las administraciones competentes y/o las mismas asociaciones hoteleras. Por último, la aplicación de bonificaciones, incentivos y ayudas económicas podrían sin duda alguna aumentar el nivel de tecnificación de aquellos hoteles que disponen de pocas o ninguna medida de ahorro hídrico avanzada.

Finalmente, destacar que el 31 de marzo de 2014, el Ayuntamiento de Lloret de Mar, el Departamento de Empresa y Empleo de la Generalitat de Cataluña y la Mesa del Turismo de Lloret de Mar firmaron un convenio de colaboración para desplegar el Plan Estratégico de Turismo de Cataluña 2013-2016. Concretamente, la localidad ha sido elegida como destino turístico maduro que es, a fin de analizar y replantear su futuro, avanzar en su modernización e innovación, con el objetivo de contribuir a la desestacionalización y el desarrollo estratégico municipal mediante un plan piloto. Para ello, se desplegarán 7 ámbitos de actuación: (1) rehabilitación y renovación de la planta de alojamiento turístico, (2) mejora del tejido urbano y económico municipal, (3) avanzar y hacer de Lloret una Smart Destination, (4) promoción de nuevas inversiones en el sector, (5) desarrollo de nuevos productos turísticos de alto valor añadido, (6) generación de nuevos eventos para enriquecer el atractivo turístico del municipio y (7) mejorar la movilidad del turista. En este sentido, y de acuerdo con los ámbitos 1 y 3 , los resultados del presente estudio pueden contribuir a la mejora de la eficiencia hídrica de la planta hotelera a partir de la transferencia de conocimientos entre los distintos grupos de hoteles que se han identificado. El concepto de Lloret de Mar Smart Destination que persigue el plan piloto es una gran oportunidad para aunar en la implementación de mecanismos y dispositivos con los que conseguir un menor consumo de agua y contribuir a una mayor resiliencia del sector turístico municipal respecto a posibles nuevos escenarios climáticos y cambios en los patrones de disponibilidad hídrica.

\section{REFERENCIAS}

ALEXANDER, S. y KENNEDY, C. (2002): «Green hotels: Opportunities and resources for success». Zero Waste Alliance, 2-9.

ANTAKYALI, D., KRAMPE, J. y STEINMETZ, H. (2008): «Practical application of wastewater reuse in tourist resorts», Water Science \& Technology, 57(12), 2051. http:// doi.org/10.2166/wst.2008.334

AYUSO, S. (2007): «Comparing voluntary policy instruments for sustainable tourism: The experience of the Spanish hotel sector», Journal of Sustainable Tourism, 15(2), 144-159. http://doi.org/10.2167/jost617.0

BARBERÁN, R., EGEA, P., GRACIA-DE-RENTERÍA, P. y SALVADOR, M. (2013): «Evaluation of water saving measures in hotels: A Spanish case study», International Journal of Hospitality Management, 34, 181-191. http://doi.org/10.1016/j.ijhm.2013.02.005 
BERRY, S. y LADKIN, A. (1997): «Sustainable tourism: a regional perspective», Tourism Management, 1S(7), 433-440. http://doi.org/10.1016/S0261-5177(97)00053-8

BOHDANOWICZ, P. (2005): «European Hoteliers' Environmental Attitudes: Greening the Business», Cornell Hotel and Restaurant Administration Quarterly, 46(2), 188204. http://doi.org/10.1177/0010880404273891

BOHDANOWICZ, P. (2005): «European hoteliers' environmental attitudes: Greening the business», Cornell Hotel and Restaurant Administration Quarterly, 46(2), 188-204. http://doi.org/10.1177/0010880404273891

BOHDANOWICZ, P. y MARTINAC,I. (2007): «Determinants and benchmarking of resource consumption in hotels - Case study of Hilton International and Scandic in Europe», Energy and Buildings, 39, 82-95. http://doi.org/10.1016/j.enbuild.2006.05.005

BRAMWELL, B. y ALLETORP, L. (2001): «Attitudes in the Danish tourism industry to the roles of business and government in sustainable tourism», International Journal of Tourism Research, 3 (2), 91-103. http://doi.org/10.1002/jtr.242

BUTLER, R.W. (2006): Tourism area life cycle: Conceptual and theoretical issues, Vol. 2. Clevedon, UK: Channel View Publications.

CARLSEN, J., GETZ, D. y ALI-KNIGHT, J. (2001): «The environmental attitudes and practices of family businesses in the rural tourism and hospitality sectors», Journal of Sustainable Tourism, 9(4), 281-297. http://doi.org/10.1080/09669580108667403

CHAN, E.S.W. (2008): «Barriers to EMS in the hotel industry», International Journal of Hospitality Management, 27 (2), 187-196. http://doi.org/10.1016/j.ijhm.2007.07.011

CHAN, E.S.W. y HAWKINS, R. (2010): «Attitude towards EMSs in an international hotel: An exploratory case study», International Journal of Hospitality Management, 29(4), 641-651. http://doi.org/10.1016/j.ijhm.2009.12.002

CHAN, E.S.W. y WONG, S.C.K. (2006): «Motivations for ISO 14001 in the hotel industry», Tourism Management, 27 (3), 481-492. http://doi.org/10.1016/j.tourman.2004.10.007

CHARARA, N., CASHMAN, A., BONNELL, R. y GEHR, R. (2011): «Water use efficiency in the hotel sector of Barbados», Journal of Sustainable Tourism, 19 (2), 231-245. http://doi.org/10.1080/09669582.2010.502577

COLE, S. (2012): «A political ecology of water equity and tourism», Annals of Tourism Research, 39(2), 1221-1241. http://doi.org/10.1016/j.annals.2012.01.003

DEYÀ, B. y TIRADO, D. (2011): «Hotel water consumption at a seasonal mass tourist destination. The case of the island of Mallorca», Journal of Environmental Management, 92, 2568-2579. http://doi.org/10.1016/j.jenvman.2011.05.024

DIPIETRO, R.B., CAO, Y. y PARTLOW, C. (2013): «Green practices in upscale foodservice operations Customer perceptions and purchase intentions», International Journal of Contemporary Hospitality Management, 25 (5), 779-796. http://doi.org/10.1108/ IJCHM-May-2012-0082

DOGC (Diari Oficial de la Generalitat de Catalunya) (2010): Decreto 183/2010, de 23 de noviembre, de establecimientos de alojamiento turístico, DOGC No. 5764, 26 de noviembre de 2010. (en catalán).

FORSYTH, T. (1997): «Environmental responsibility and business regulation: the case of sustainable tourism», The Geogrpahical Journal, 163 (3), 270-280. Recuperado de http://www.blackwellpublishing.com/journals/geoj/ 
FUENTES, J.L. (2003): Técnicas de riego. Madrid: Ediciones Mundi-Prensa.

GABARDA-MALLORQUÍ, A. y RIBAS, A. (2016): «Understanding reductions in water consumption in tourist areas: a case study of the Costa Brava, Spain», International Journal of Water Resources Development, 32 (6), 912-930. http://doi.org/10.1080/07 900627.2016.1142861

GABARDA-MALLORQUI, A., GARCIA, X. y RIBAS, A. (2017): «Mass tourism and water efficiency in the hotel industry. A case study», International Journal of Hospitality Management, 61, 82-93.

GATT, K. y SCHRANZ, C. (2015): «Retrofitting a 3 star hotel as a basis for piloting water minimisation interventions in the hospitality sector», International Journal of Hospitality Management, 50, 115-121. http://doi.org/10.1016/j.ijhm.2015.06.008

GÖSSLING, S. (2001): «The consequences of tourism for sustainable water use on a tropical island: Zanzibar, Tanzania», Journal of Environmental Management, 61, 179-91. http://doi.org/10.1006/jema.2000.0403

GÖSSLING, S., PEETERS, P., HALL, C.M., CERON, J.P., DUBOIS, G., LEHMANN, L.V. y SCOTT, D. (2012): «Tourism and water use: Supply, demand, and security. An international review», Tourism Management, 33, 1-15. http://doi.org/10.1016/j. tourman.2011.03.015

HADJIKAKOU, M., CHENOWETH, J. y MILLER, G. (2013): «Estimating the direct and indirect water use of tourism in the eastern Mediterranean», Journal of Environmental Management, 114, 548-556. http://doi.org/10.1016/j.jenvman.2012.11.002

HILLARY, R. (2004): «Environmental management systems and the smaller enterprise», Journal of Cleaner Production, 12 (6), 561-569. http://doi.org/10.1016/j.jclepro.2003.08.006

HILLS, S., BIRKS, R. y MCKENZIE, B. (2002): «The Millennium Dome "Watercycle" experiment: To evaluate water efficiency and customer perception at a recycling scheme for 6 million visitors», Water Science and Technology, 46, 233-240.

HJALAGER, A.M. (1998): «Environmental regulation of tourism: Impact on business innovation», Progree in Tourism and Hospitality Research, 4, 17-30.

HOF, A. y SCHMITT, T. (2011): «Urban and tourist land use patterns and water consumption: Evidence from Mallorca, Balearic Islands», Land Use Policy, 28, 792-804. http:// doi.org/10.1016/j.landusepol.2011.01.007.

INSTITUTO NACIONAL DE ESTADÍSTICA (2013): Cuenta Satélite del Turismo de España. Base 2008. Serie 2008-2012.

JIMÉNEZ, G. (2011): Estudio comparativo entre condensación por aire y por agua para la refrigeración del edifico Juan Benet. Universidad Carlos III de Madrid.

KASIM, A., GURSOY, D., OKUMUS, F. y WONG, A. (2014): «The importance of water management in hotels: a framework for sustainability through innovation», Journal of Sustainable Tourism, 22(7), 1090-1107. http://doi.org/10.1080/09669582.2013.873444

KENT, M., NEWNHAM, R. y ESSEX, S. (2002): «Tourism and sustainable water supply in Mallorca: a geographical analysis», Applied Geography, 22, 351-374. http://doi. org/10.1016/S0143-6228(02)00050-4

KIRK, D. (1998): «Attitudes to environmental management held by a group of hotel managers in Edinburgh», International Journal of Hospitality Management, 17(1), 33-47. http://doi.org/10.1016/S0278-4319(98)00005-X 
KNOWLES, T., MACMILLAN, S., PALMER, J., GRABOWSKI, P. y HASHIMOTO, A. (1999): «The development of environmental initiatives in tourism: responses from the London hotel sector», International Journal of Tourism Research, 1 (4), 255-265. http:// doi.org/10.1002/(SICI)1522-1970(199907/08)1:4<255::AID-JTR170>3.0.CO;2-8

MANCO, D. G., ERAZO, J. G. y OCAMPO, A.M. (2012): «Eficiencia en el consumo de agua de uso residencial», Revista Ingenierías Universidad de Medellín, 11 (21), 23-38. Recuperado de http://datateca.unad.edu.co/contenidos/358029/2014/Unidad_2/ a2._Manco_D_Ahorro_Agua_Residencial.pdf

MOROTE, Á. y HERNÁNDEZ, M. (2014): «Jardines y urbanizaciones, nuevas naturalezas urbanas en el litoral de la provincia de Alicante», Documents d'Anàlisi Geogràfica, 60 (3), 483-504.

NYAHUNZVI, D.K. (2013): «CSR reporting among Zimbabwe's hotel groups: a content analysis», International Journal of Contemporary Hospitality Management, 25 (4), 595-613. http://doi.org/10.1108/09596111311322943

O'CONNOR, R.E., BORD, R.J., YARNAL, B. y WIEFEK, N. (2002): «Who wants to reduce greenhouse gas emissions?», Social Science Quarterly, 83(1), 1-17. http://doi. org/10.1111/1540-6237.00067

PADULLÉS, J. (2015): Socioeconomic status determines floristic patterns in suburban domestic gardens: implications for water use and alien plant dispersal in the Mediterranean context (tesis doctoral). Universidad de Girona, Girona.

PADULLÉS, J., VILA, J. y BARRIOCANAL, C. (2014): «Maintenance, modifications, and water use in private gardens of Alt Empordà, Spain», Horttechnology, 24 (3), 374-383.

PADULLÉS, J., VILA, J. y BARRIOCANAL, C. (2015): «Propagule pressure from invasive plant species in gardens in low-density suburban areas of the Costa Brava (Spain)», Urban Forestry and Urban Greening, 14 (4), 941-951. http://doi.org/10.1016/j.ufug.2015.09.002

PARÉS, M., DOMENE, E. y SAURÍ, D. (2004): «Gestión del agua en la jardinería pública y privada de la región metropolitana de Barcelona», Boletín de la Asociación de Geógrafos Españoles, 37, 223-238.

QUAZI, H. a, KHOO, Y., TAN, C. y WONG, P. (2001): «Motivation for ISO 14000 certification: development of a predictive model», The International Journal of Management Science, 29, 525-542. http://doi.org/10.1016/S0305-0483(01)00042-1

R DEVELOPMENT CORE TEAM (RDCT) (2010): $R$ : A language and environment for statistical computing. Recuperado de http://www.R-project.org/

RANDOLPH, B. y TROY, P. (2008): «Attitudes to conservation and water consumption», Environmental Science and Policy, 11, 441-455. http://doi.org/10.1016/j. envsci.2008.03.003

RICO-AMORÓS, A. M., SAURÍ, D., OLCINA-CANTOS, J. y VERA-REBOLLO, J.F. (2013): «Beyond megaprojects? Water alternatives for mass tourism in coastal mediterranean Spain», Water Resources Management, 27, 553-565. http://doi.org/10.1007/ s11269-012-0201-3

SMITH, M., HARGROVES, K., DESHA, C. y STASINOPOULOS, P. (2009): Water transformed - Australia: sustainable water solutions for climate change adaptation. Recuperado de http://www.naturaledgeproject.net/Sustainable_Water_Solutions_Portfolio.aspx 
SORIANO, A. (2010): Instalaciones de agua para un consumo sostenible. Recuperado de http://www.grados.com.es/sites/default/files/Instalaciones_agua_consumo_sostenible[1].pdf

STABLER, M.J. y GOODALL, B. (1997): «Environmental awareness, action and performance in the Guernsey hospitality sector», Tourism Management, 18 (1), 19-33. http:// doi.org/10.1016/S0261-5177(96)00095-7

STYLES, D., SCHOENBERGER, H. y GALVEZ-MARTOS, J.L. (2015): «Water management in the European hospitality sector: Best practice, performance benchmarks and improvement potential», Tourism Management, 46, 187-202. http://doi.org/10.1016/j. tourman.2014.07.005

TZSCHENTKE, N., KIRK, D. y LYNCH, P.A. (2004): «Reasons for going green in serviced accommodation establishments», International Journal of Contemporary Hospitality Management, 16 (2), 116-124. http://doi.org/10.1108/09596110410520007

VERNON, J., ESSEX, S., PINDER, D. y CURRY, K. (2003): «The "greening" of tourism micro-businesses: Outcomes of focus group investigations in South East Cornwall», Business Strategy and the Environment, 12, 49-69. http://doi.org/10.1002/bse.348

VIDERAS, J. y ALBERINI, A. (2000): «The appeal of voluntary environmental programs: Which firms participate and why?", Contemporary Economic Policy, 18 (4), 449-461. http://doi.org/10.1111/j.1465-7287.2000.tb00041.x 